\title{
Chance-Constrained Stochastic Programming under Variable Reliability Levels with an Application to Humanitarian Relief Network Design
}

\author{
Özgün Elçi, Nilay Noyan ${ }^{1}$, Kerem Bülbül \\ Industrial Engineering Program, Sabancı University, 34956 Istanbul, Turkey \\ ozgunelci@sabanciuniv.edu, nnoyan@sabanciuniv.edu, bulbul@sabanciuniv.edu
}

January 21, 2018

\begin{abstract}
We focus on optimization models involving individual chance constraints, in which only the right-hand side vector is random with a finite distribution. A recently introduced class of such models treats the reliability levels / risk tolerances associated with the chance constraints as decision variables and trades off the actual cost / return against the cost of the selected reliability levels in the objective function. Leveraging recent methodological advances for modeling and solving chance-constrained linear programs with fixed reliability levels, we develop strong mixed-integer programming formulations for this new variant with variable reliability levels. In addition, we introduce an alternate cost function type associated with the risk tolerances which requires capturing the value-at-risk (VaR) associated with a variable reliability level. We accomplish this task via a new integer linear programming representation of VaR. Our computational study illustrates the effectiveness of our mathematical programming formulations. We also apply the proposed modeling approach to a new stochastic last mile relief network design problem and provide numerical results for a case study based on the real-world data from the 2011 Van earthquake in Turkey.
\end{abstract}

Keywords: stochastic programming; risk-averse; probabilistic constraints; chance constraints; variable reliability; Bonferroni approximation; value-at-risk; network design; post-disaster; humanitarian logistics; accessibility; equity

1. Introduction In many decision making problems under uncertainty it is desired to account for the probability of certain unfavorable events. This is very much in agreement with the concept of reliability often used to characterize the quality of service. In a wide range of domains, applications concerned with such issues (the probability of meeting demand or the reliability of a system) give rise to mathematical programming models that involve chance (or probabilistic) constraints. Areas of application of chance-constrained optimization models include but are not restricted to production planning, supply chain management, power system planning and design, financial portfolio optimization, and humanitarian relief network design. For a thorough overview of the applications of chance-constrained optimization models and the corresponding theory and numerical methods, we refer the reader to Kall and Wallace (1994), Prékopa (1995), Prékopa (2003), Dentcheva (2006), Shapiro et al. (2009), and the references therein.

There are two main types of chance constraints: joint and individual (separate) chance constraints. A joint chance constraint imposes that a set of goal constraints (inequalities) hold together with a high probability. In contrast, an individual chance constraint is introduced to account for the probability of a single goal constraint. From a modeling point of view, the problem of interest determines the appropriate type of chance constraint. As discussed in Haneveld and van der Vlerk (2015), a joint chance constraint is more fitting when the individual goal constraints collectively describe one single goal. Otherwise, if the individual goal constraints describe different goals, it makes more sense to consider them separately. In this case, the ability to vary the reliability levels associated with the separate chance constraints provides us with a flexible modeling framework, which can prioritize the set of goals at hand. In practice, another important criterion is the computational tractability of the resulting mathematical programming formulations. Optimization with a joint chance constraint is generally significantly more challenging than optimization with individual chance constraints (see, e.g., Luedtke et al., 2010; Küçükyavuz, 2012; Lejeune, 2012). Enforcing a joint chance constraint with a high probability level on a large set of goal constraints typically leads to individual probability levels close to one, and consequently, may result in very conservative solutions. As a partial remedy, the decision maker may opt for decreasing the probability level of the joint chance constraint, but this exacerbates the computational difficulties. Therefore, one may prefer to employ individual chance constraints even if a joint chance constraint is more appropriate from a modeling perspective. In particular, it is natural to use individual chance constraints to develop a 
computationally tractable approximation of a joint chance-constrained optimization model (see, e.g., Prékopa, 2003; Haneveld and van der Vlerk, 2015).

Our study is dedicated to individual chance-constrained linear programs (LP), where the uncertainty is restricted to the right-hand sides of the probabilistic constraints, and the random parameters have discrete distributions. The convexity of the feasible region is still preserved in this structure given the fixed probability levels. A general form of the classical LP with multiple individual chance constraints is then given by:

(MICC)

Here, the feasible set associated with the deterministic constraints is represented by the polyhedron $\mathcal{X} \subseteq \mathbb{R}_{+}^{n}$. We assume that $T$ is a deterministic $m \times n$ matrix with its $k$ th row denoted by $T_{k}$, and $\boldsymbol{\xi}=\left(\xi_{1}, \ldots, \xi_{m}\right)^{T}$ is an $m$-dimensional random vector with a finite support. The realizations of the random vector $\boldsymbol{\xi}$ are denoted by $\boldsymbol{\xi}^{i}$ with corresponding probabilities $p^{i}>0$ for $i \in\{1, \ldots, N\}$. The individual chance constraints (2) ensure that the stochastic (goal) constraint $T_{k} \mathbf{x} \geq \xi_{k}$ holds with a probability at least equal to $1-\epsilon_{k}$ for all $k \in\{1, \ldots, m\}$, where $\epsilon_{k}$ is the risk tolerance corresponding to the reliability level $1-\epsilon_{k}$. Note that integrality requirements can also be incorporated into the definition of $\mathcal{X}$, and our entire modeling and solution framework directly carries over to individual chance-constrained integer linear programs as well.

It is well-known that (MICC) can be reformulated as a linear program when the risk tolerances $\epsilon_{k}, k=$ $1, \ldots, m$, are input parameters specified by the decision maker. Alternatively, we can consider the risk tolerances / reliability levels as decision variables, and the resulting mathematical models can be put into good use in several ways at the expense of additional complexity. For instance, (MICC) with a set of additional constraints on the variable/adjustable reliability levels is presented in Prékopa (2003) - see formulation (5.10) - as an approximation of a joint chance-constrained optimization model. This approximation optimizes the risk tolerances and is a natural extension of the classical Bonferroni approximation, which is based on the particular choice of equal risk tolerances. Another motivation for varying the values of the reliability levels is to perform a Pareto analysis in order to extract insights about the trade-off between the actual cost/return factors and the cost associated with the probabilities of the undesirable events of interest (Rengarajan and Morton, 2009; Rengarajan et al., 2013). In a similar vein, Shen (2014) proposes a new class of optimization models with adjustable reliability levels, where the author incorporates a linear cost function of the individual risk tolerances into the objective function (1). Lejeune and Shen (2016) follow this line of research in the context of joint chance-constrained optimization. They consider two types of a joint constraint - with a deterministic technology matrix $T$ on the left-hand side (randomness exists only on the right-hand side) and with a random technology matrix $T$ and develop effective mathematical programming formulations based on a Boolean modeling framework. We also refer to Lejeune and Shen (2016) for a detailed review on studies which consider a trade-off between the conflicting cost/return criteria and reliability objectives.

Our study is directly related to and motivated by Shen (2014). The author presents a mixed-integer linear programming (MIP) reformulation for (MICC) with adjustable risk tolerances. However, the reformulation of the chance constraints relies on the classical big- $M$ paradigm and solving large instances presents a formidable challenge due to the well-known weakness of the LP relaxations of formulations featuring big- $M$ s. Our primary objective in this paper is therefore to offer a computationally effective MIP reformulation of (MICC) when the reliability levels are treated as decision variables. To this end, we exploit recent methodological advances for modeling and solving chance-constrained linear programs with fixed reliability levels. In particular, we use a modeling approach similar to that presented in Luedtke et al. (2010). The fundamental idea is to rewrite the chance constraints (2) in the form of $T_{k} \mathbf{x} \geq z_{k}$, where $z_{k}$ corresponds to the $\left(1-\epsilon_{k}\right)$ th quantile of the random component $\xi_{k}$ for $k \in\{1, \ldots, m\}$. Variable reliability levels render the quantile values denoted by $z_{k}, k \in\{1, \ldots, m\}$, variable as well, and reformulating the chance constraints requires being able to express the 
quantile values as functions of the reliability level variables. To this end, we develop two alternate approaches to express the decision variables $z_{k}, k \in\{1, \ldots, m\}$. The first representation is based on the mixing inequalities proposed by Luedtke et al. (2010). The authors study the mixing set with a knapsack constraint arising in the deterministic equivalent formulation of a joint chance-constrained optimization model with a finitely distributed random right-hand side and a fixed reliability level. It turns out that the results of this work can be applied to individual chance-constrained optimization models with adjustable risk tolerances as well. An alternate second representation arises from using a different set of binary variables to identify the scenarios under which the goal constraints are violated. The resulting MIP formulations scale very well with an increasing number of scenarios and outperform the current state-of-the-art based on the big- $M$ type of constraints by a significant margin - see Section 5. Therefore, one noteworthy contribution of our work is to highlight the existence and efficacy of alternate formulations for individual chance-constrained (integer) linear programs with and without variable risk tolerances and make recent methodological progress in modeling and solving chance-constrained optimization models more accessible to practitioners.

Optimization capturing the trade-off between the actual cost factors and the cost of the risk tolerances associated with the chance constraints is a fairly recent research area, and such a hybrid modeling approach has promise to be applied in different fields. In this context, we elaborate on how to construct a cost function of the variable reliability levels and extend/modify Shen (2014)'s model by quantifying the cost of reliability with a different perspective. Ultimately, we apply the proposed modeling approach to humanitarian relief logistics, where it may be essential to consider multiple and possibly conflicting performance criteria - such as accessibility and equity, see, e.g., Noyan et al. (2016). In particular, we focus on balancing the tradeoff between accessibility and the level of demand satisfaction in the context of post-disaster relief network design. We introduce a new stochastic last mile distribution network design problem, which determines the locations of the Points of Distribution (PODs), the assignments of the demand nodes to PODs, and the delivery amounts to the demand nodes while considering the equity and accessibility issues and incorporating the inherent uncertainties. The studies that consider decisions related to the locations of the last mile facilities are scarce, and as emphasized in Noyan et al. (2016), the majority of these studies either assume a deterministic setting and/or do not incorporate the concepts of accessibility and equity. Our study contributes to the humanitarian relief literature by introducing a new hybrid supply allocation policy and developing a new risk-averse optimization model, which is well-solved with the proposed MIP formulations. We delay the relevant literature review on relief network design until Section 4.

The rest of the paper is organized as follows. In Section 2, we present two effective alternate MIP formulations of (MICC) with adjustable/variable reliability levels. In Section 3, we extend these formulations to a related class of models that focus on balancing the trade-off between the cost/return and the reliability levels. Section 4 is dedicated to the new stochastic last mile distribution network design problem discussed above. This is followed in Section 5 by the computational study, while Section 6 contains the concluding remarks.

2. Optimization Models with Individual Chance Constraints In this section, we first present the classical mathematical programming reformulation of (MICC) with fixed reliability levels, and then delve into the extensions of (MICC) with variable risk tolerances. Before we proceed, some of the conventions used throughout the paper are due here. $F_{X}$ designates the cumulative distribution function of a random variable $X$. The set of the first $n$ positive integers is denoted by $[n]=\{1, \ldots, n\}$, while $[a]_{+}=\max (a, 0)$ indicates the positive part of a number $a \in \mathbb{R}$.

Quantiles play a major part in the reformulations of chance-constrained optimization models, where the first quantile function $F_{X}^{(-1)}:(0,1] \rightarrow \mathbb{R}$ of a random variable $X$ is the generalized inverse of the cumulative distribution $F_{X}$ given by $F_{X}^{(-1)}(\alpha)=\inf \left\{\eta \in \mathbb{R}: F_{X}(\eta) \geq \alpha\right\}$. This quantity is also known as the value-at-risk (VaR) of the random variable $X$ at confidence level $\alpha \in(0,1]$ and denoted by $\operatorname{VaR}_{\alpha}(X)$. Observing that the 
equivalence relation

$$
\mathbb{P}\left(T_{k} \mathbf{x} \geq \xi_{k}\right) \geq 1-\epsilon_{k} \quad \Leftrightarrow \quad T_{k} \mathbf{x} \geq F_{\xi_{k}}^{(-1)}\left(1-\epsilon_{k}\right),
$$

holds for any $k \in[m]$ yields the following LP as the deterministic equivalent formulation of (MICC) with fixed risk tolerances $\epsilon_{k}, k \in[m]$ :

$$
\begin{aligned}
(\mathbf{M I C C}-\mathbf{D E F}) \quad \min _{\mathbf{x}} & \mathbf{c}^{T} \mathbf{x} \\
\text { s.t. } & T_{k} \mathbf{x} \geq F_{\xi_{k}}^{(-1)}\left(1-\epsilon_{k}\right), \quad \forall k \in[m] \\
& \mathbf{x} \in \mathcal{X} .
\end{aligned}
$$

The inequalities in (6) are linear because the quantile values $F_{\xi_{k}}^{(-1)}\left(1-\epsilon_{k}\right), k \in[m]$, are input parameters calculated a priori for the given set of scenarios representing the distribution of the random vector $\boldsymbol{\xi}$. For instance, in our humanitarian relief network design problem of Section $4, \xi_{k}$ designates the random demand at some location $k$ and the value $\operatorname{VaR}_{1-\epsilon_{k}}\left(\xi_{k}\right)$ specifies a lower bound on the total supply delivered to this location that is exceeded with a large probability $1-\epsilon_{k}$. Since we enforce individual chance constraints, without loss of generality we can assume that the realizations of $\xi_{k}$ are relabeled so that $\xi_{k}^{1} \geq \cdots \geq \xi_{k}^{N}$ holds with the corresponding probabilities $p_{k}^{i}, i=1, \ldots, N$, for all $k \in[m]$. Then, it is easy to see that we have $F_{\xi_{k}}^{(-1)}(\alpha)=\xi_{k}^{i_{k}^{\prime}}$ for all $k \in[m]$, where

$$
i_{k}^{\prime}:=\max \left\{i \in[N]: \sum_{l=1}^{i} p_{k}^{l} \leq 1-\alpha\right\}+1=\max \left\{i \in[N]: \sum_{l=i}^{N} p_{k}^{l} \geq \alpha\right\} .
$$

As discussed in Section 1, one may prefer to allow the reliability levels to be decision variables in some decision making problems. Consequently, the quantile values are also incorporated as decision variables into this version of (MICC) - referred to as (MICC - VR - DEF) in this paper. It turns out that optimizing over a set of individual chance constraints with variable reliability levels is strongly $\mathcal{N} \mathcal{P}$-hard (Theorem 8, Xie et al., 2017). In this case, we cannot preserve an LP structure and have to resort to the binary variables $\beta_{k i}, k \in[m], i \in[N]$, so that $\beta_{k i}$ is set to one if $T_{k} \mathbf{x}<\xi_{k}^{i}$ - as ensured by the constraints (9b) in the formulation below:

$$
\begin{array}{rlr}
(\mathbf{M I C C}-\mathbf{V R}-\mathbf{D E F}) & \min _{\mathbf{x}, \boldsymbol{\epsilon}, \boldsymbol{\beta}} \mathbf{c}^{T} \mathbf{x} & \\
\text { s.t. } & T_{k} \mathbf{x} \geq \xi_{k}^{i}-M \beta_{k i}, & \forall k \in[m], i \in[N] \\
& \sum_{i \in[N]} p_{k}^{i} \beta_{k i} \leq \epsilon_{k}, & \\
& A \epsilon \leq \mathbf{b}, & \\
& 0 \leq \epsilon_{k} \leq \bar{\epsilon}_{k}, & \\
& \mathbf{x} \in \mathcal{X}, & \\
& \boldsymbol{\beta} \in\{0,1\}^{m \times N} . &
\end{array}
$$

The constraints (9b)-(9c), where $M$ is a large number, collectively mandate that $\mathbb{P}\left(T_{k} \mathbf{x} \geq \xi_{k}\right) \geq 1-\epsilon_{k}$. Given an optimal solution $(\hat{\mathbf{x}}, \hat{\boldsymbol{\beta}}, \hat{\boldsymbol{\epsilon}})$, we have $T_{k} \hat{\mathbf{x}} \geq \max \left\{\xi_{k}^{i}: i \in[N], \hat{\beta}_{k i}=0\right\}$ for $k \in[m]$. It is easy to see that the right-hand side of this expression is the quantile value in (6) for the risk tolerance $\epsilon_{k}=\sum_{i \in[N]: \hat{\beta}_{k i}=1} p_{k}^{i}$. Two types of constraints are imposed on the variable risk tolerances $\epsilon_{k}, k \in[m]$. The reliability level of an individual chance constraint $k \in[m]$ can be no less than $1-\bar{\epsilon}_{k}$ as ensured by the simple upper bound constraints (9e). In addition, we include a general set of constraints (9d) with $\boldsymbol{\epsilon}=\left(\epsilon_{1}, \ldots, \epsilon_{m}\right)^{T}$ on the variable risk tolerances. In applications - say in a production planning problem or in a humanitarian relief context such as that in Section 4, constraints (9d) may reflect the relative importance of an individual chance constraint with respect to the others. To illustrate, if the population center 1 (node 1) in a humanitarian relief network design problem is deemed to be more critical than population center 2 (node 2) based on its characteristics, then the constraint $a_{12} \epsilon_{1} \leq \epsilon_{2}$ with $a_{12}>1$ would be included in the formulation. This would mandate a higher level of demand 
satisfaction at population center 1 compared to that at population center 2. A similar interpretation equally applies to a production planning problem by substituting customers for population centers. On the other hand, if (MICC - VR - DEF) is employed as an approximation of a corresponding optimization model with an embedded joint chance constraint of the form $\mathbb{P}\left(T_{k} \mathbf{x} \geq \xi_{k}, k \in[m]\right) \geq \alpha$ as discussed in the introduction, then (9d) consists of a single constraint $\sum_{k \in[m]} \epsilon_{k} \leq 1-\alpha$ and also implies that $\bar{\epsilon}_{k}=1-\alpha$ for all $k \in[m]$ in (9e). In contrast, the traditional Bonferroni approximation sets $\epsilon_{k}=(1-\alpha) / m$ for all $k \in[m]$. Here, allowing variable reliability levels is of essence to improve the effectiveness of the Bonferroni approximation. Such an approximation can for instance be used in order to solve a humanitarian relief network design model incorporating a joint chance constraint with the goal of satisfying the demand across the network with a specified high probability. This probability may be labeled as the minimum required level of "network-wide reliability" (see, e.g., Hong et al., 2015).

A MIP formulation of the form (MICC - VR - DEF) quickly becomes computationally intractable as the number of scenarios increases due to the presence of the big- $M$ type of constraints - such as (9b). The key result, which enables stronger and computationally effective formulations based on recent methodological progress, is that for a given $k \in[m]$, the set of constraints $T_{k} \mathbf{x} \geq \xi_{k}^{i}-M \beta_{k i}, i \in[N]$, can be substituted by a single constraint

$$
T_{k} \mathbf{x} \geq z_{k}
$$

where $z_{k}$ is a quantile expressed purely through the realizations $\xi_{k}^{i}, i=1, \ldots, N$, and a set of binary variables. This result rests on the equivalence relation (4) and the fact that the realizations of $\boldsymbol{\xi}$ can be sorted independently for each component $k \in[m]$ because we deal with individual chance constraints. These two observations together imply the existence of a threshold index $i_{k}^{\prime}$ for any fixed set of decisions $\overline{\mathbf{x}}$ such that $T_{k} \overline{\mathbf{x}}<\xi_{k}^{i}$ for $i=1, \ldots i_{k}^{\prime}-1$, and $T_{k} \overline{\mathbf{x}} \geq \xi_{k}^{i}$ for $i=i_{k}^{\prime}, \ldots N$. Correspondingly, the values of the associated $\beta$-variables are set as $\beta_{k i}=1$ for $i=1, \ldots i_{k}^{\prime}-1$, and $\beta_{k i}=0$ for $i=i_{k}^{\prime}, \ldots N$. Exploiting this structure and detecting the correct value of $i_{k}^{\prime}$ is the central theme in the development of the valid inequalities and variable reduction techniques summarized in the next lemma. The proofs are omitted either because they are simple to derive or exist in the references provided.

\section{LEMMA 2.1}

i. Preprocessing - variable reduction (see, e.g., Luedtke et al., 2010; Lejeune and Noyan, 2010). Let $i_{k}^{*}:=\max \left\{i \in[N]: \sum_{l=1}^{i} p_{k}^{l} \leq \bar{\epsilon}_{k}\right\}+1$ for $k \in[m]$. Then, the necessary condition for any $\mathbf{x} \in \mathcal{X}$ to satisfy (2) with $\epsilon_{k} \leq \bar{\epsilon}_{k}, k \in[m]$, is that the following quantile-based inequalities hold:

$$
T_{k} \mathbf{x} \geq \xi_{k}^{i_{k}^{*}} \quad \forall k \in[m] .
$$

Consequently, $\beta_{k i}=0$ for all $k \in[m], i \in\left\{i_{k}^{*}, \ldots, N\right\}$, in any feasible solution of (MICC - VR - DEF), and these variables can be omitted from the formulation. Note that $\bar{\epsilon}_{k}$ is an input parameter while $\epsilon_{k}$ is a decision variable, and by the definition of $i_{k}^{*}$ we have $F_{k}^{(-1)}\left(1-\bar{\epsilon}_{k}\right)=\xi_{k}^{i_{k}^{*}} \geq \xi_{k}^{i}$ for all $i \in\left\{i_{k}^{*}, \ldots, N\right\}$. Then, by the monotonicity of the first quantile function, $F_{k}^{(-1)}\left(1-\epsilon_{k}\right) \geq F_{k}^{(-1)}\left(1-\bar{\epsilon}_{k}\right)$ holds, and the assertion follows from (4).

ii. Valid inequalities (see, e.g., Ruszczyński, 2002; Luedtke et al., 2010). The following inequalities are valid for (MICC - VR - DEF):

$$
\beta_{k i} \geq \beta_{k, i+1} \quad \forall k \in[m], i \in[N-1] .
$$

iii. For a discrete random variable $\xi_{k}$ with realizations $\xi_{k}^{1} \geq \cdots \geq \xi_{k}^{N}$ and corresponding probabilities $p_{k}^{i}, i \in[N]$, $\operatorname{VaR}_{\lambda}\left(\xi_{k}\right)=\operatorname{VaR}_{\alpha}\left(\xi_{k}\right)$ holds for any $\lambda \in\left(\alpha_{-}, \alpha_{+}\right]$, where $\alpha_{-}=\mathbb{P}\left(\xi_{k}<\operatorname{VaR}_{\alpha}\left(\xi_{k}\right)\right)$ and $\alpha_{+}=\mathbb{P}\left(\xi_{k} \leq\right.$ $\left.\operatorname{VaR}_{\alpha}\left(\xi_{k}\right)\right)$. In other words, the quantile function $F_{k}^{(-1)}$ in (4) is constant on the following intervals:

$$
F_{k}^{(-1)}(\alpha)=\xi_{k}^{i} \quad \forall \alpha \in\left(\sum_{l=i+1}^{N} p_{k}^{l}, \sum_{l=i}^{N} p_{k}^{l}\right], k \in[m] .
$$


iv. Valid equalities. Based on item (iii), there exists an optimal solution to (MICC - VR - DEF) for finite probability spaces such that the variable $\epsilon_{k}$ is set to one of the values $\left\{\tilde{p}_{k}^{i_{k}^{*}-1}, \tilde{p}_{k}^{i_{k}^{*}-2}, \cdots, \tilde{p}_{k}^{1}, \tilde{p}_{k}^{0}\right\}$, where $\tilde{p}_{k}^{i}=\mathbb{P}\left(\xi_{k} \geq \xi_{k}^{i}\right)=\sum_{l \in[i]} p_{k}^{l}$ for $i \in\left[i_{k}^{*}-1\right]$ and $\tilde{p}_{k}^{0}=0$. Then, the following constraints are valid for $($ MICC - VR - DEF $)$ :

$$
\epsilon_{k}=\sum_{i \in\left[i_{k}^{*}-1\right]} \tilde{p}_{k}^{i}\left(\beta_{k i}-\beta_{k, i+1}\right) \text { with } \beta_{k, i_{k}^{*}}=0 \quad \forall k \in[m] .
$$

A similar set of constraints featuring a different type of binary variables is used in Shen (2014).

v. Strengthened star (mixing) inequalities (Luedtke et al., 2010). The big-M constraints (9b) in (MICC - VR - DEF) can be substituted by

$$
T_{k} \mathbf{x} \geq \xi_{k}^{1}-\sum_{i \in\left[i_{k}^{*}-1\right]}\left(\xi_{k}^{i}-\xi_{k}^{i+1}\right) \beta_{k i}, \quad \forall k \in[m] .
$$

Starting from the structure of the $\beta$-variables discussed preceding Lemma 2.1, it is straightforward to attach an intuitive meaning to the inequalities (12). In order to explain the theoretical basis of these valid inequalities, observe that the random vector $\boldsymbol{\xi} \in \mathbb{R}^{m}$ can be assumed to be non-negative without loss of generality - see Section 2 in Luedtke et al. (2010). This trivially implies that $T_{k} \mathbf{x} \geq 0$ for every feasible solution given any set of reasonable reliability values (i.e, $\epsilon_{k}<1, k \in[m]$ ), and we can replace (9b) by $T_{k} \mathbf{x} \geq \xi_{k}^{i}\left(1-\beta_{k i}\right), \forall k \in$ $[m], i \in[N]$. This new form of the big- $M$ constraints exposes the connection with the mixing set - a well-known concept in integer programming. This connection is exploited by Luedtke et al. (2010), who focus on a single chance constraint indexed by $k \in[m]$ with a fixed risk tolerance level $\bar{\epsilon}_{k}$ as a means of developing strong MIP formulations for joint chance-constrained LPs. The authors first leverage the knapsack inequality (9c) to obtain a strengthening of the set $G_{k}=\left\{\left(y, \boldsymbol{\beta}_{k}\right) \in \mathbb{R}_{+} \times\{0,1\}^{N}: \sum_{i \in[N]} p_{k}^{i} \beta_{k i} \leq \bar{\epsilon}_{k}, y+\xi_{k}^{i} \beta_{k i} \geq \xi_{k}^{i}, i \in[N]\right\}$ associated with the inequalities $T_{k} \mathbf{x} \geq \xi_{k}^{i}\left(1-\beta_{k i}\right), \forall i \in[N]$. Then, they apply the mixing inequalities of Günlük and Pochet (2001) to establish that (12) are facet-defining for $G_{k}$; however, (12) does not characterize the convex hull. The validity of these inequalities for (MICC - VR - DEF) can be argued from the necessary condition (6) and the relation $F_{\xi_{k}}^{(-1)}\left(1-\epsilon_{k}\right) \geq F_{\xi_{k}}^{(-1)}\left(1-\bar{\epsilon}_{k}\right)$ implied by the upper bounding constraints (9e). Embedding facet-defining inequalities into MIP formulations yields tighter LP relaxations and quicker solution times. Such enhanced formulations are commonly labeled as "strong" formulations, and we follow suit by marking the strong formulations presented in the rest of the paper with the letter "S" appended to the head of the formulation titles.

At the fundamental level, all enhancements that enable the strong formulations in this paper rely on sorting the realizations of the random right-hand side vector in the goal constraints independently for each $k \in[m]$. Therefore, it is essential for us that randomness is restricted to the right-hand side of the goal constraints. However, if randomness is present in the technology matrix $T$ on the left-hand side - as in the setting of Lejeune and Shen (2016), then we can resort to a generalization and extension of the mixing set concepts developed in Luedtke (2014).

Recall that the end goal in our endeavor to arrive at a stronger alternative to (MICC $-\mathbf{V R}-\mathbf{D E F})$ is to replace the set of constraints $T_{k} \mathbf{x} \geq \xi_{k}^{i}-M \beta_{k i}, i \in[N]$, by a single constraint (10). The main challenge we face in this task is to express $z_{k}=F_{\xi_{k}}^{(-1)}\left(1-\epsilon_{k}\right)=\operatorname{VaR}_{\left(1-\epsilon_{k}\right)}\left(\xi_{k}\right)$ via linear constraints, where $\epsilon_{k}$ is a decision variable. To this end, we can build upon Lemma 2.1 and use one of the linear representations of VaR provided in the next lemma, which - to the best of our knowledge - have not appeared in the literature before. An alternate representation of VaR has been proposed in Küçükyavuz and Noyan (2016); however, that one is more general and is also valid when the realizations cannot be sorted in advance, e.g., when the outcomes depend on the decisions. Ultimately, formulations incorporating this more general representation of VaR are more complicated. In this paper, we can enjoy the simpler representations of Lemma 2.2 for the special case when the realizations can be ordered a priori. 
Lemma 2.2 Suppose that $V$ is a random variable with realizations $v^{1} \geq \ldots \geq v^{N}$ and corresponding probabilities $p^{i}>0, i \in[N]$. Let $\tilde{p}^{i}=\mathbb{P}\left(V \geq v^{i}\right)=\sum_{l \in[i]} p^{l}$ and $i^{*}:=\max \left\{i \in[N]: \tilde{p}^{i} \leq \bar{\epsilon}\right\}+1$, where $\bar{\epsilon}$ is a positive constant in $(0,1]$. Then, for a given risk tolerance $\epsilon^{\prime}$ such that $0 \leq \epsilon^{\prime} \leq \bar{\epsilon}$, the equality $\operatorname{VaR}_{\left(1-\epsilon^{\prime}\right)}(V)=z^{\prime}$ holds if and only if

(i) there exists a vector $\left(z, \epsilon, \beta_{0}, \boldsymbol{\beta}\right)$ satisfying

$$
\begin{aligned}
& z=v^{1}-\sum_{i \in\left[i^{*}-1\right]}\left(v^{i}-v^{i+1}\right) \beta_{i}, \\
& \sum_{i \in\left[i^{*}-1\right]} p^{i} \beta_{i} \leq \epsilon, \\
& \sum_{i \in\left[i^{*}-1\right]} p^{i} \beta_{i}+\sum_{i \in\left[i^{*}\right]}\left(\beta_{i-1}-\beta_{i}\right) p^{i} \geq \epsilon+\lambda, \\
& \beta_{0}=1, \\
& \beta_{i^{*}}=0 \\
& \beta_{i} \geq \beta_{i+1}, \\
& \beta_{i} \in\{0,1\}, \\
& 0 \leq \epsilon \leq \bar{\epsilon},
\end{aligned}
$$

where $\boldsymbol{\beta} \in\{0,1\}^{i^{*}}, z=z^{\prime}$, and $\lambda$ is a sufficiently small positive constant to ensure that the constraint (13c) is equivalent to the strict inequality $\sum_{i \in\left[i^{*}-1\right]} p^{i} \beta_{i}+\sum_{i \in\left[i^{*}\right]}\left(\beta_{i-1}-\beta_{i}\right) p^{i}>\epsilon$.

(ii) there exists a vector $(z, \epsilon, \boldsymbol{\beta})$ satisfying

$$
\begin{aligned}
& \sum_{i \in\left[i^{*}-1\right]}\left(\beta_{i}-\beta_{i+1}\right) \tilde{p}^{i}=\epsilon, \\
& (13 \mathrm{a}),(13 \mathrm{e})-(13 \mathrm{~h}),
\end{aligned}
$$

where $\boldsymbol{\beta} \in\{0,1\}^{i^{*}}$ and $z=z^{\prime}$.

Proof. We distinguish between two cases. First, assume that $0 \leq \epsilon^{\prime}<p^{1}$ which implies $\operatorname{VaR}_{\left(1-\epsilon^{\prime}\right)}(V)=$ $v^{1}$. In this case, it is a simple matter to verify that $\left(z, \epsilon, \beta_{0}, \boldsymbol{\beta}\right)=\left(v^{1}, \epsilon^{\prime}, 1,(0, \ldots, 0)\right)$ and $(z, \epsilon, \boldsymbol{\beta})=$ $\left(v^{1}, 0,(0, \ldots, 0)\right)$ are feasible with respect to (13) and (14), respectively. Conversely, the existence of a feasible solution $\left(z, \epsilon, \beta_{0}, \boldsymbol{\beta}\right)=\left(v^{1}, \epsilon^{\prime}, 1,(0, \ldots, 0)\right)$ with $0 \leq \epsilon^{\prime}<p_{1}$ for (13) correctly implies that $\operatorname{VaR}_{\left(1-\epsilon^{\prime}\right)}(V)=v^{1}$. On the other hand, a feasible solution $(z, \epsilon, \boldsymbol{\beta})=\left(v^{1}, 0,(0, \ldots, 0)\right)$ of $(14)$ maps back to $\operatorname{VaR}_{\left(1-\epsilon^{\prime}\right)}(V)=v^{1}$ for any $0 \leq \epsilon^{\prime}<p^{1}$ by Lemma 2.1-(iii).

If $p^{1} \leq \epsilon^{\prime} \leq \bar{\epsilon}$, then $\operatorname{VaR}_{\left(1-\epsilon^{\prime}\right)}(V)=v^{\ell+1}$ for some $\ell \in\left[i^{*}-1\right]$. For this case, we can construct two feasible solutions $\left(z, \epsilon, \beta_{0}, \boldsymbol{\beta}\right)=\left(v^{\ell+1}, \epsilon^{\prime}, 1,(1,1, \ldots, 1,0, \ldots, 0)\right)$ and $(z, \epsilon, \boldsymbol{\beta})=\left(v^{\ell+1}, \tilde{p}^{\ell},(1,1, \ldots, 1,0, \ldots, 0)\right)$ for (13) and (14), respectively. In both cases, $\boldsymbol{\beta}$ is composed of ones in the first $\ell$ positions, followed by all zeros for the remaining components. To check the feasibility of these solutions, note that $\boldsymbol{\beta}$ fulfills (13e)(13g) and inserting it into (13a) yields $z=v^{\ell+1}$. In addition, the constraints (13b)-(13d) prescribe that $\sum_{i \in[\ell]} p^{i} \leq \epsilon^{\prime}<\sum_{i \in[\ell+1]} p^{i}$. This must hold when $\operatorname{VaR}_{\left(1-\epsilon^{\prime}\right)}(V)=v^{\ell+1}$, and therefore, the first solution is feasible for (13). To establish the feasibility of the second solution for (14), observe that $\epsilon=\tilde{p}^{\ell}$ satisfies (14a), and (13h) is ensured by $\ell \leq i^{*}-1$ and the definition of $i^{*}$. Starting from the same feasible solutions, we can show the converse of these statements and complete the proof through arguments analogous to those in the first part of the proof.

The illustration in the example below helps explain how the system (13) expresses $\operatorname{VaR}_{\left(1-\epsilon^{\prime}\right)}$. 
EXAMPLE 2.1 Suppose that for a given confidence level $1-\epsilon^{\prime}$ such that $0 \leq \epsilon^{\prime} \leq \bar{\epsilon}, \operatorname{VaR}_{\left(1-\epsilon^{\prime}\right)}(V)=v^{\ell+1}$ for some $\ell \in\left[i^{*}-1\right]$ and $\operatorname{VaR}_{(1-\bar{\epsilon})}(V)=v^{i^{*}}$.

$$
\begin{aligned}
& \overbrace{v^{1} \geq \cdots \geq v^{\ell} \geq v^{\ell+1}}^{\sum_{i \in[\ell+1]} p^{i}>\epsilon^{\prime}} \geq \cdots \geq v^{i^{*}} \geq \cdots \geq v^{N} \\
& \underbrace{v_{\operatorname{VaR}_{\left(1-\epsilon^{\prime}\right)}(V)}}_{\sum_{i \in[\ell]} p^{i} \leq \epsilon^{\prime}} \underbrace{v^{\ell+1}}
\end{aligned}
$$

According to (13b)-(13g), $\beta_{i}=1$ for $i \leq \ell$ (realizations in red) and $\beta_{i}=0$ for $i \geq \ell+1$ (realizations in blue).

Then, (13a) takes the following form:

$$
\begin{aligned}
z & =v^{1}-\left(v^{1}-v^{2}\right) \beta_{1}-\left(v^{2}-v^{3}\right) \beta_{2}-\cdots-\left(v^{\ell}-v^{\ell+1}\right) \beta_{\ell} \\
& =v^{1}-\left(v^{1}-v^{2}\right)-\left(v^{2}-v^{3}\right)-\cdots-\left(v^{\ell}-v^{\ell+1}\right)=v^{\ell+1}
\end{aligned}
$$

Thus, as desired, we ensure that $z=\operatorname{VaR}_{\left(1-\epsilon^{\prime}\right)}(V)=v^{\ell+1}$.

As is also evident from the proof of Lemma 2.2, (13) is developed starting from the first expression in (8). On the other hand, the underlying premise of (14) is Lemma 2.1-(iii), which allows us to substitute (13b)-(13d) with (14a) to obtain (14) from (13). This observation further asserts that (14) is a stronger representation of $\operatorname{VaR}_{(1-\epsilon)}(V)$ compared to (13) because all feasible solutions of (13) of the form $\left(z, \epsilon, \beta_{0}, \boldsymbol{\beta}\right)=\left(v^{\ell+1}, \epsilon^{\prime}, 1,(1,1, \ldots, 1,0, \ldots, 0)\right)$ with $\tilde{p}^{\ell} \leq \epsilon^{\prime}<\tilde{p}^{\ell+1}$ correspond to a single feasible solution $(z, \epsilon, \boldsymbol{\beta})=\left(v^{\ell+1}, \tilde{p}^{\ell},(1,1, \ldots, 1,0, \ldots, 0)\right)$ of (14). Finally, employing the enhancements of Lemma 2.1 and the VaR representation (14), we arrive at a stronger MIP formulation of individual chance-constrained LPs with variable reliability levels given below. An alternate formulation based on the VaR representation (13) can be obtained in a very similar fashion.

$$
\begin{array}{rlr}
(\mathbf{S M I C C}-\mathbf{V R}-\boldsymbol{\beta}) \quad \min _{\mathbf{x}, \mathbf{z}, \boldsymbol{\epsilon}, \boldsymbol{\beta}} & \mathbf{c}^{T} \mathbf{x} & \\
\text { s.t. } & T_{k} \mathbf{x} \geq z_{k}, & \\
& z_{k}=\xi_{k}^{1}-\sum_{i \in\left[i_{k}^{*}-1\right]}\left(\xi_{k}^{i}-\xi_{k}^{i+1}\right) \beta_{k i}, & \forall k \in[m] \\
& \beta_{k i} \geq \beta_{k, i+1}, & \forall k \in[m] \\
& \beta_{k, i_{k}^{*}}=0, & \forall k \in[m], i \in\left[i_{k}^{*}-2\right] \\
& \epsilon_{k}=\sum_{i \in\left[i_{k}^{*}-1\right]} \tilde{p}_{k}^{i}\left(\beta_{k i}-\beta_{k, i+1}\right), & \forall k \in[m] \\
& (9 \mathrm{~d})-(9 \mathrm{f}), & \forall k \in[m] \\
& \beta_{k i} \in\{0,1\}, & \forall k \in[m], i \in\left[i_{k}^{*}\right] .
\end{array}
$$

For risk-averse decision makers, the typical values for the risk tolerances are small values such as 0.05 and implies that the reasonable choices for the values of the corresponding upper bounds $\bar{\epsilon}_{k}$ would also be small. Therefore, the variable reduction-based preprocessing methods are very effective in reducing the number of binary variables and constraints. However, in general, even the reduced form of (MICC - VR - DEF) may not scale well with increasing problem size. The proposed formulations featuring the quantile-based representation in the form of (10) have a huge positive impact on the solution times and the maximum instance size that can be handled effectively. These claims will be substantiated in Section 5.2.

We conclude this section by providing an alternate equivalent formulation of (SMICC $-\mathbf{V R}-\boldsymbol{\beta})$ based on a different set of binary variables. To this end, we define $y_{k i} \in\{0,1\}, k \in[m], i \in[N]$, so that $y_{k i}$ takes the value of 1 if $T_{k} \mathbf{x}<\xi_{k}^{i-1}$ and $T_{k} \mathbf{x} \geq \xi_{k}^{i}$ hold together. Clearly, we have $y_{k i}=\beta_{k, i-1}-\beta_{k i}$ with the understanding that $\beta_{k 0}=1$. In this notation, exactly one of the variables $y_{k i}, i \in\left[i_{k}^{*}\right],-$ say $y_{k i^{\prime}}$ - assumes the value 1 for 
each $k \in[m]$ as mandated by the constraints (16e) below. Accordingly, the variable risk tolerance $\epsilon_{k}$ and the corresponding quantile $z_{k}$ are set to $\tilde{p}_{k}^{i^{\prime}-1}=\sum_{l \in\left[i^{\prime}-1\right]} p_{k}^{l}$ and $\xi_{k}^{i^{\prime}}$ by the constraints (16d) and (16c), respectively. This VaR representation (16c)-(16f) expressed via the $y$-variables provides us with the formulation stated next:

$$
\begin{array}{rlr}
\min _{\mathbf{x}, \mathbf{z}, \mathbf{\epsilon}, \mathbf{y}} & \mathbf{c}^{T} \mathbf{x} & \\
\text { s.t. } & T_{k} \mathbf{x} \geq z_{k}, & \\
& z_{k}=\sum_{i \in\left[i_{k}^{*}\right]} \xi_{k}^{i} y_{k i}, & \forall k \in[m] \\
& \epsilon_{k}=\sum_{i \in\left[i_{k}^{*}\right]} \tilde{p}_{k}^{i-1} y_{k i}, & \forall k \in[m] \\
& \sum_{i \in\left[i_{k}^{*}\right]} y_{k i}=1, & \forall k \in[m] \\
& y_{k i} \in\{0,1\}, & \forall k \in[m] \\
& (9 \mathrm{~d})-(9 \mathrm{f}) . & \forall k \in[m], i \in\left[i_{k}^{*}\right]
\end{array}
$$

Shen (2014) bases her formulations on these $y$-variables - as will be explained in more depth in the next section. Our motivation for providing this alternate formulation is to form a unified framework for the models introduced in the rest of the paper and a foundation for discussing Shen (2014)'s model in the next section. Finally, we point out that the LP relaxations of $(\mathbf{S M I C C}-\mathbf{V R}-\boldsymbol{\beta})$ and $(\mathbf{S M I C C}-\mathbf{V R}-\mathbf{y})$ are equivalent; however, these two formulations do not necessarily perform similarly in our computational experiments because the different cuts generated by the solver based on the $\beta$ - and $y$-variables lead to different search trees.

3. Balancing the Actual Return/Cost and Risk In this section, we focus on a class of optimization models with variable reliability levels, where the objective function also features a cost term associated with the variable risk tolerances. The aim is to make decisions by taking into account the trade-off between the actual cost/return and risk. We first present such an existing model proposed by Shen (2014) and provide associated strong MIP formulations based on the modeling constructs of Section 2, which prove to be effective in solving large problem instances in Section 5. In the second part of this section, we take a different stance from Shen (2014) in capturing the trade-off between the variable reliability levels and the actual cost/return and introduce an alternate way of quantifying the cost of reliability in the objective. A corresponding new class of optimization models with effective MIP formulations are presented.

3.1 Underlying Optimization Model A general form of the extended version of (MICC - VR - DEF), which incorporates the trade-off between the actual cost/return and risk is given by

$$
\begin{aligned}
& (\mathbf{M I C C}-\text { VRT }) \quad \min _{\mathbf{x}, \boldsymbol{\epsilon}} \mathbf{c}^{T} \mathbf{x}+\sum_{k \in[m]} h_{k}\left(\epsilon_{k}\right) \\
& \text { s.t. }(2),(9 \mathrm{~d})-(9 \mathrm{f}) \text {. }
\end{aligned}
$$

In this modeling approach, the characterization of the cost function $h_{k}:\left[0, \bar{\epsilon}_{k}\right] \rightarrow \mathbb{R}$ plays a fundamental role. Shen (2014) considers a monotonically increasing linear function of the form $h_{k}\left(\epsilon_{k}\right)=a_{k} \epsilon_{k}$ with $a_{k}>0$ for all $k \in[m]$. The author justifies her model by pointing out several problem settings with conflicting individual chance constraints, where it may make sense to enable the decision maker to adjust the individual reliability levels within their respective allowable intervals by solving (MICC - VRT). In a way, this model is akin to multi-criteria methods, which form a single composite objective function by taking a weighted sum of the individual conflicting objectives, and presents an alternative to exploring the Pareto frontier by iteratively solving one of appropriate formulations from Section 2 with manually adjusted reliability levels. The issue 
whether a linear function of the risk tolerances incorporated directly into the objective - as in (17) - serves the intended purpose is taken up in the next section. We first present the deterministic equivalent formulation of (MICC - VRT) proposed by Shen (2014), which employs the $y$ - variables introduced at the end of Section 2. Compared to Shen (2014)'s original formulation, the version below is slightly enhanced by incorporating the preprocessing technique of Lemma 2.1-(i).

$$
\begin{aligned}
&(\mathbf{M I C C}-\mathbf{V R T}-\mathbf{D E F}) \quad \min _{\mathbf{x}, \boldsymbol{\epsilon}, \mathbf{y}} \mathbf{c}^{T} \mathbf{x}+\sum_{k \in[m]} a_{k} \epsilon_{k} \\
& \text { subject to } \quad T_{k} \mathbf{x} \geq \xi_{k}^{i} y_{k i}-M\left(1-y_{k i}\right), \quad \forall k \in[m], i \in\left[i_{k}^{*}\right] \\
& \sum_{k \in[m]} \epsilon_{k} \leq \Gamma \\
&(9 \mathrm{f}),(16 \mathrm{~d})-(16 \mathrm{f}) .
\end{aligned}
$$

Obviously, the underlying premise of this formulation is the well-known quantile-based inequality (4) and the observation formalized in Lemma 2.1-(iii). The simple upper bounds on the variable risk tolerances - see (9e) - are excluded and the generic restrictions (9d) are replaced by a single constraint (19c) in this formulation, where $\Gamma$ is a given budget of risk shared by all chance constraints. The big- $M$ constraints (19b) enforce that $T_{k} \mathbf{x} \geq \xi_{k}^{i} y_{k i}$ whenever $y_{k i}=1$ and can be substituted by the enhanced form $T_{k} \mathbf{x} \geq \xi_{k}^{i} y_{k i}$ by relying on the non-negativity of the random right-hand side vector $\boldsymbol{\xi} \in \mathbb{R}^{m}$ without loss of generality - see the discussion immediately following Lemma 2.1. This latter form is also recognized - but not put to use - by Shen (2014) and is utilized in our computational study, but it turns out that even the pre-processed reduced formulation (MICC - VRT - DEF) does not scale well as the problem size increases. In particular, Shen (2014) employs (MICC - VRT - DEF) to solve a multi-commodity flow network capacity design problem with a pretty limited number of - three or six - individual chance constraints. The number of scenarios is kept constant throughout, and the results indicate that the computational effort expended tends to grow sharply with increasing network size and larger values of $\Gamma$. In her concluding remarks, Shen (2014) points out that iterative methods relying on repeatedly solving (MICC) with fixed reliability levels is so far not promising for tackling (MICC - VRT) and emphasizes the need for good MIP formulations to this end. We take up this issue in this paper and develop substantially more effective and scalable MIP formulations for (MICC - VRT) by drawing upon the modeling tools of Section 2. We illustrate our claims in Section 5 by performing computational tests on two classes of problems: a transportation problem (Luedtke et al., 2010) and the stochastic network design problem described in Section 4.

Compared to (SMICC-VR-y) in the previous section, the drawback of the formulation (MICC - VRT - DEF) is that it fails to insert sufficiently large quantile values into the right-hand sides of the constraints (19b). The crucial factor here is calculating the quantile values correctly as a function of the variable risk levels $\epsilon_{k}, k \in[m]$, and representing the inequalities (19b) compactly. This issue is also of essential significance in Section 3.2, where we devise an alternate expression for capturing the cost of reliability in the objective function. Ultimately, we obtain a strong formulation of (MICC - VRT) by appending the term $\sum_{k \in[m]} a_{k} \epsilon_{k}$ to the objective function of (SMICC $-\mathbf{V R}-\mathbf{y})$. This formulation is referred to as (SMICC $-\mathbf{V R T}-\mathbf{y})$ in the rest of the paper. In a similar vein, incorporating the same term $\sum_{k \in[m]} a_{k} \epsilon_{k}$ into the objective function of $(\mathbf{S M I C C}-\mathbf{V R}-\boldsymbol{\beta})$ yields an alternate strong formulation $(\mathbf{S M I C C}-\mathbf{V R T}-\boldsymbol{\beta})$ based on the $\beta$-variables. The numerical results in Section 5 attest to the significant computational edge of (SMICC $-\mathbf{V R T}-\mathbf{y})$ and $($ SMICC - VRT $-\boldsymbol{\beta})$ over $($ MICC - VRT - DEF $)$ - with gains of up to two orders of magnitude in solution times for some instances. These experimental outcomes highlight the recent advances in the field of stochastic programming (see, e.g., Luedtke et al., 2010; Küçükyavuz, 2012) and confirm their potential applicability to practical problems, in which the uncertainty is captured with a large number of scenarios.

3.2 A New Approach to Quantifying the Cost of Reliability Chance constraints are based on a qualitative risk concept and measure the probabilities of violating the stochastic constraints, irrespective of 
the magnitude of violation. Shen (2014)'s approach of incorporating the term $\sum_{k \in[m]} a_{k} \epsilon_{k}$ into the objective function may be considered as an attempt at constructing a hybrid stochastic model with both qualitative and quantitative aspects. We concur with this perspective and also quote from Prékopa (1995), who notes that "the best model construction is the one which combines the use of a probabilistic constraint and penalties in the objective function," as a further motivating support from the literature. However, we find that a linear function of the risk tolerances directly appended to the objective function falls short of properly capturing the cost of reliability for two reasons. First, the two components of the objective function (19a) are not commensurate; i.e., they are not on the same scale. Second, and more importantly, the structure of (19a) implies that decreasing the value of $\epsilon_{k}$ from 0.10 to 0.09 and from 0.05 to 0.04 have the same impact of $0.01 a_{k}$ on the reliability component of the objective function value. This is hardly justifiable as the first quantile function is not linear in the risk tolerance. To provide a concrete example, suppose that decreasing the risk tolerance from 0.10 to 0.09 requires supplying an additional 50 units of a particular product, while decreasing the risk tolerance from 0.05 to 0.04 may require an additional supply of 200 items. Clearly, the cost function associated with reliability should not treat these two cases identically. Ultimately, we contend that in order to account for the nonlinear structure of the first quantile function, the cost coefficient $a_{k}$ in (19a) should be specified according to the level of the quantity-based service associated with a particular risk tolerance. Observe that according to the relation (4), changing the value of the risk tolerance $\epsilon_{k}$ is equivalent to changing the right-hand side of the corresponding constraint in the deterministic equivalent formulation - see, e.g., (16b)-(16d). From this viewpoint, it is more natural to define the cost function associated with reliability explicitly in terms of the associated VaR values. More specifically, we suggest to quantify the cost of reliability with a function of the form $h_{k}\left(\operatorname{VaR}_{\left(1-\epsilon_{k}\right)}\left(\xi_{k}\right)\right)$, which leads to the problem statement:

$$
\text { (MICC - VRTQ) }
$$

$$
\begin{aligned}
\min _{\mathbf{x}, \boldsymbol{\epsilon}} & \mathbf{c}^{T} \mathbf{x}+\sum_{k \in[m]} h_{k}\left(\operatorname{VaR}_{\left(1-\epsilon_{k}\right)}\left(\xi_{k}\right)\right) \\
& (2),(9 \mathrm{~d})-(9 \mathrm{f}) .
\end{aligned}
$$

In this context, larger VaR values - equivalently, smaller $\epsilon_{k}$ values - are preferred in terms of the reliability levels of the chance constraints. Consequently, we consider monotonically decreasing cost functions $h_{k}()$ in order to incorporate the cost of compromising from the reliability levels. The particular focus is on functions that can be represented by linear objective terms and constraints.

Due to the variability of the reliability levels, the main task in reformulating (MICC - VRTQ) as a MIP is to express $F_{\xi_{k}}^{(-1)}\left(1-\epsilon_{k}\right)=\operatorname{VaR}_{\left(1-\epsilon_{k}\right)}\left(\xi_{k}\right)$ via linear constraints and can be accomplished by drawing upon the modeling tools of Section 2. A MIP formulation of (MICC - VRTQ) is obtained from $(\mathbf{S M I C C}-\mathbf{V R T}-\mathbf{y})$ in a straightforward way:

$$
\begin{aligned}
(\mathbf{S M I C C}-\mathbf{V R T Q}-\mathbf{y}) \quad \min _{\mathbf{x}, \mathbf{z}, \boldsymbol{\epsilon}, \mathbf{y}} & \mathbf{c}^{T} \mathbf{x}+\sum_{k \in[m]} h_{k}\left(z_{k}\right) \\
\text { s.t. } & (16 \mathrm{~b})-(16 \mathrm{~g}) .
\end{aligned}
$$

We can also develop alternate MIP formulations employing the $\beta$ - variables in the spirit of Sections 2 and 3.1 . As underlined previously at the end of Section 2, equivalent formulations based on the $y-$ and $\beta$-variables are closely related, but they may still exhibit different computational behavior. From this perspective, it makes sense to extend (SMICC - VRT - $\boldsymbol{\beta})$ by integrating the new cost functions $h_{k}\left(\operatorname{VaR}_{\left(1-\epsilon_{k}\right)}\left(\xi_{k}\right)\right), k \in[m]$. To this end, we can put either one of the VaR representations in Lemma 2.2 to use. The presentation (14) was previously demonstrated in $(\mathbf{S M I C C}-\mathbf{V R}-\boldsymbol{\beta})$. To illustrate the use of (13), here we only present the MIP formulation of (MICC - VRTQ) obtained from (SMICC - VRT - $\boldsymbol{\beta}$ ) by incorporating (13):

$$
\begin{aligned}
& (\mathbf{S M I C C}-\mathbf{V R T Q}-\boldsymbol{\beta}) \quad \min _{\mathbf{x}, \mathbf{z}, \boldsymbol{\epsilon}, \boldsymbol{\beta}} \mathbf{c}^{T} \mathbf{x}+\sum_{k \in[m]} h_{k}\left(z_{k}\right) \\
& \text { s.t. }(15 \mathrm{~b})-(15 \mathrm{e}),(15 \mathrm{~g})-(15 \mathrm{~h}) \text {, } \\
& \sum_{i \in\left[i_{k}^{*}-1\right]} p_{k}^{i} \beta_{k i} \leq \epsilon_{k}, \quad \forall k \in[m]
\end{aligned}
$$




$$
\begin{array}{ll}
\sum_{i \in\left[i_{k}^{*}-1\right]} p_{k}^{i} \beta_{k i}+\sum_{i \in\left[i_{k}^{*}\right]}\left(\beta_{k, i-1}-\beta_{k i}\right) p_{k}^{i} \geq \epsilon_{k}+\lambda, & \forall k \in[m] \\
\beta_{k 0}=1, & \forall k \in[m] .
\end{array}
$$

An important aspect of the VaR representations in Lemma 2.2 is that they capture the value of $\mathrm{VaR}_{(1-\epsilon)}$ precisely without relying on the structure of the objective function. This is essential given that the second component of the objective function of (MICC - VRTQ) related to the cost of reliability favors larger values of VaR. Otherwise, if smaller quantile values would be preferred by the objective, then (13c) could safely be omitted from the representation (13).

We next elaborate on our particular choice of the cost function $h_{k}\left(\operatorname{VaR}_{\left(1-\epsilon_{k}\right)}\left(\xi_{k}\right)\right)$. One option is to set $h_{k}\left(\operatorname{VaR}_{\left(1-\epsilon_{k}\right)}\left(\xi_{k}\right)\right)=-a_{k} \operatorname{VaR}_{\left(1-\epsilon_{k}\right)}\left(\xi_{k}\right)$ with $a_{k}>0$. Alternatively, we define it based on the random outcome $\left[\xi_{k}-\operatorname{VaR}_{\left(1-\epsilon_{k}\right)}\left(\xi_{k}\right)\right]_{+}$, which allows us to incorporate a quantitative measure on the potential violation of the corresponding stochastic goal constraint. As noted at the start of this section, such hybrid approaches are promoted in the literature as good modeling practice (Prékopa, 1995). In this spirit, we intend to determine the optimal risk tolerance $\epsilon_{k}$ by also taking into account the realizations of $\xi_{k}$ in excess of the lower threshold value $\operatorname{VaR}_{\left(1-\epsilon_{k}\right)}\left(\xi_{k}\right)$. In particular, we focus on the expected violation and set $h_{k}\left(\operatorname{VaR}_{\left(1-\epsilon_{k}\right)}\left(\xi_{k}\right)\right)=a_{k} E\left(\left[\xi_{k}-\right.\right.$ $\left.\left.\operatorname{VaR}_{\left(1-\epsilon_{k}\right)}\left(\xi_{k}\right)\right]_{+}\right)$. If scenario-dependent cost coefficients are required or preferred, then the cost function takes the form of

$$
h_{k}\left(\operatorname{VaR}_{\left(1-\epsilon_{k}\right)}\left(\xi_{k}\right)\right)=\sum_{i \in\left[i i_{k}^{*}-1\right]} a_{k i} p_{k}^{i}\left[\xi_{k}^{i}-\operatorname{VaR}_{\left(1-\epsilon_{k}\right)}\left(\xi_{k}\right)\right]_{+} .
$$

For this choice of quantifying the cost of reliability, we define $w_{k i}$ to represent $\left[\xi_{k}^{i}-\operatorname{VaR}_{\left(1-\epsilon_{k}\right)}\left(\xi_{k}\right)\right]_{+} \cdot \operatorname{Then}$,

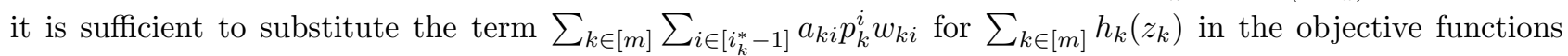
of (SMICC - VRTQ - y) and (SMICC - VRTQ - $\boldsymbol{\beta})$ and append the following two constraints to the formulation in either case:

$$
\begin{array}{ll}
w_{k i} \geq \xi_{k}^{i}-z_{k}, & \forall k \in[m], i \in\left[i_{k}^{*}-1\right] \\
w_{k i} \geq 0, & \forall k \in[m], i \in\left[i_{k}^{*}-1\right] .
\end{array}
$$

The proposed approach is a flexible modeling tool to balance the trade-off between the actual cost/return and the cost of reliability and accounts for the violation of the stochastic goal constraints both qualitatively and quantitatively.

For ease of reference, we provide a summary of the compact MIP formulations of the presented chanceconstrained optimization models in Table 1. These models are not domain-specific and may be applied to a wide spectrum of problems, where factoring in the probability of uncertain unfavorable events is essential to meet the reliability requirements of the system under design - see the first paragraph of Section 1 . Incorporating this concern into an optimization model has primarily been accomplished through chance-constraints with fixed reliability levels up until recently. Along with recent research, we take this modeling approach one notch further in this paper and provide the reader with an enhanced modeling construct that can explicitly account for the trade-off between the cost of reliability and the reliability levels selected for different stochastic goal constraints. Ultimately, however, we emphasize that the particular type of chance-constrained model to be used - with either fixed or variable reliability levels - depends on the specific application and is up to the decision maker. Our purpose here is to expose the availability of advanced formulation concepts in chance-constrained optimization and enhance the modeling toolbox of the decision maker without necessarily expressing a strong preference for one particular type of model. The next section puts some of the proposed models with variable reliability levels into use for humanitarian relief network design; however, the applicability of our modeling framework certainly extends to other domains. Specific existing relevant examples include multi-commodity flow network capacity design (Shen, 2014) and multi-portfolio financial optimization (Lejeune and Shen, 2016).

On the one hand, the appropriate model is application- and context-dependent, and it is the decision maker's prerogative to select the model to apply - as underlined above. On the other hand, a couple of high-level 
fundamental considerations weigh into the choice of the correct chance-constrained optimization model with variable reliability levels and its associated parameters. For instance, in the approximation of a joint chanceconstrained problem - see Section 1, the cost of reliability is irrelevant, and one of the formulations with variable reliability levels in Section 2 must be used. In contrast, in our humanitarian relief network design problem in Section 4, demand shortages matter, and therefore, we follow the modeling approach of Section 3.2 to explicitly penalize the violations of the goal constraints in the objective. Another principal issue is identified as a consequence of the insights gleaned from the computational study in Section 5.3: the results are sensitive to the values of $\bar{\epsilon}_{k}, \forall k \in[m]$, in the constraints (9e) as well as to the values of the set of parameters $a$, which appear in the objective function of the models presented in Section 3 as part of the cost of reliability. A decision maker not comfortable with identifying the values of these central parameters may resort to a Pareto-type approach as outlined at the end of Section 5.3. Regardless of the domain of application, such key factors and considerations should be integrated into the modeling process of chance-constraints with variable reliability levels.

\begin{tabular}{|c|c|}
\hline Model & MIP Formulation \\
\hline $\begin{array}{l}(\mathbf{S M I C C}-\mathbf{V R}-\boldsymbol{\beta}) \\
\text { Based on } \beta \text {-variables }\end{array}$ & $\min _{\mathbf{x}, \mathbf{z}, \boldsymbol{\epsilon}, \boldsymbol{\beta}}\left\{\mathbf{c}^{T} \mathbf{x}:(15 \mathrm{~b})-(15 \mathrm{~h})\right\}$, or simply, (15) \\
\hline $\begin{array}{l}(\mathbf{S M I C C}-\mathbf{V R}-\mathbf{y}) \\
\text { Based on } y \text {-variables }\end{array}$ & $\min _{\mathbf{x}, \mathbf{z}, \epsilon, \mathbf{y}}\left\{\mathbf{c}^{T} \mathbf{x}:(16 \mathrm{~b})-(16 \mathrm{~g})\right\}$, or simply, $(16)$ \\
\hline $\begin{array}{l}\text { (MICC - VRT - DEF }) \\
\text { Shen (2014)'s model }\end{array}$ & $\min _{\mathbf{x}, \boldsymbol{\epsilon}, \mathbf{y}}\left\{\mathbf{c}^{T} \mathbf{x}+\sum_{k \in[m]} a_{k} \epsilon_{k}:(19 \mathrm{~b})-(19 \mathrm{~d})\right\}$, or simply, (19) \\
\hline $\begin{array}{c}(\mathbf{S M I C C}-\mathbf{V R T}-\mathbf{y}) \\
(\mathbf{S M I C C}-\mathbf{V R}-\mathbf{y}) \text { with trade-off }\end{array}$ & $\min _{\mathbf{x}, \mathbf{z}, \boldsymbol{\epsilon}, \mathbf{y}}\left\{\mathbf{c}^{T} \mathbf{x}+\sum_{k \in[m]} a_{k} \epsilon_{k}:(16 \mathrm{~b})-(16 \mathrm{~g})\right\}$ \\
\hline $\begin{array}{c}\text { SMICC }- \text { VRT }-\boldsymbol{\beta}) \\
(\mathbf{S M I C C}-\mathbf{V R}-\boldsymbol{\beta}) \text { with trade-off }\end{array}$ & $\min _{\mathbf{x}, \mathbf{z}, \boldsymbol{\epsilon}, \boldsymbol{\beta}}\left\{\mathbf{c}^{T} \mathbf{x}+\sum_{k \in[m]} a_{k} \epsilon_{k}:(15 \mathrm{~b})-(15 \mathrm{~h})\right\}$ \\
\hline $\begin{array}{c}(\mathbf{S M I C C}-\mathbf{V R T Q}-\mathbf{y}) \\
(\mathbf{S M I C C}-\mathbf{V R}-\mathbf{y}) \text { with VaR-based trade-off }\end{array}$ & $\min _{\mathbf{x}, \mathbf{z}, \epsilon, \mathbf{y}}\left\{\mathbf{c}^{T} \mathbf{x}+\sum_{k \in[m]} h_{k}\left(z_{k}\right):(16 \mathrm{~b})-(16 \mathrm{~g})\right\}$, or simply, (21) \\
\hline $\begin{array}{c}(\mathbf{S M I C C}-\mathbf{V R T Q}-\boldsymbol{\beta}) \\
(\mathbf{S M I C C}-\mathbf{V R}-\boldsymbol{\beta}) \text { with VaR-based trade-off }\end{array}$ & $\min _{\mathbf{x}, \mathbf{z}, \boldsymbol{\epsilon}, \boldsymbol{\beta}}\left\{\mathbf{c}^{T} \mathbf{x}+\sum_{k \in[m]} h_{k}\left(z_{k}\right):(22 \mathrm{~b})-(22 \mathrm{e})\right\}, \quad$ or simply, (22) \\
\hline $\begin{array}{c}(\text { SMICC - VRTQA - y }) \\
(\text { SMICC }- \text { VRTQ }-\mathbf{y}) \text { with }(23)\end{array}$ & $\min _{\mathbf{x}, \mathbf{z}, \mathbf{\epsilon}, \mathbf{y}, \mathbf{w}}\left\{\mathbf{c}^{T} \mathbf{x}+\sum_{k \in[m],} a_{i \in\left[i_{k}^{*}-1\right]} a_{k i} p_{k}^{i} w_{k i}:(16 \mathrm{~b})-(16 \mathrm{~g}),(24)-(25)\right\}$ \\
\hline $\begin{array}{c}(\text { SMICC }- \text { VRTQA }-\boldsymbol{\beta}) \\
(\text { SMICC }- \text { VRTQ }-\boldsymbol{\beta}) \text { with }(23)\end{array}$ & $\min _{\mathbf{x}, \mathbf{z}, \boldsymbol{\epsilon}, \boldsymbol{\beta}, \mathbf{w}}\left\{\mathbf{c}^{T} \mathbf{x}+\sum_{k \in[m],} a_{i \in\left[i i_{k}^{*}-1\right]} a_{k i} p_{k}^{i} w_{k i}:(22 \mathrm{~b})-(22 \mathrm{e}),(24)-(25)\right\}$ \\
\hline
\end{tabular}

${ }^{1}$ : The big- $M$ constraints (19b) in Shen (2014) are substituted by the enhanced form $T_{k} \mathbf{x} \geq \xi_{k}^{i} y_{k i}, \forall k \in[m], i \in\left[i_{k}^{*}\right]$.

${ }^{2}: \operatorname{size}(\mathbf{x})=n, \operatorname{size}(\mathbf{z})=\operatorname{size}(\boldsymbol{\epsilon})=m, \operatorname{size}(\mathbf{y})=\operatorname{size}(\boldsymbol{\beta})=\sum_{k \in[m]} i_{k}^{*}, \operatorname{size}(\mathbf{w})=\sum_{k \in[m]}\left(i_{k}^{*}-1\right) ;$

$i_{k}^{*}=\left\lfloor N \bar{\epsilon}_{k}\right\rfloor+1, \forall k \in[m]$ for equal scenario probabilities.

${ }^{3}: \mathbf{y}, \boldsymbol{\beta}$ : binary; $\mathbf{z}, \boldsymbol{\epsilon}, \mathbf{w}$ : continuous; $\mathbf{x}$ : binary/continuous.

${ }^{4}:(\mathbf{S M I C C}-\mathbf{V R}-\mathbf{y}),(\mathbf{S M I C C}-\mathbf{V R T}-\mathbf{y}),(\mathbf{S M I C C}-\mathbf{V R T Q}-\mathbf{y})$ contain $\mathcal{O}(m+q+r)$ constraints, where $q$ and $r$ denote the number of constraints in (9d) and (9f), respectively. The corresponding figure for the remaining formulations is $\mathcal{O}(m N+q+r)$, except that (MICC - VRT - DEF) includes $\mathcal{O}(m N+r)$ constraints.

Table 1: Summary of the MIP formulations of the chance-constrained optimization models.

4. A Stochastic Optimization Model for Designing Last Mile Relief Networks We introduce a new stochastic last mile distribution network design problem and develop an associated stochastic optimization model that incorporates the concepts of accessibility and equity while capturing the uncertainty in post-disaster demands and transportation network conditions. The proposed model showcases the generic chance-constrained stochastic programming formulations with variable reliability levels of the previous sections.

4.1 Literature Review There is a growing body of literature devoted to the development of stochastic programming models for humanitarian relief logistics (see, e.g., Liberatore et al., 2013). The majority of 
these studies involving facility location decisions are dedicated to the pre-disaster management context (see, e.g., Balcik and Beamon, 2008; Rawls and Turnquist, 2010; Salmerón and Apte, 2010; Mete and Zabinsky, 2010; Döyen et al., 2012). Moreover, most of the existing studies - with the exception of a few (e.g., Beraldi and Bruni, 2009; Rawls and Turnquist, 2011; Noyan, 2012; Hong et al., 2015; Elçi and Noyan, 2018) - propose risk-neutral stochastic programming models. However, making decisions based on the expected values may not be good enough for rarely occurring disaster events, and it may be essential to take into consideration the random variability inherent in chaotic disaster relief systems. To the best of our knowledge, risk-averse stochastic models for post-disaster relief network design are at best scarce, and models with chance constraints are absent. Even the more extensive literature on pre-disaster relief network design includes only a few studies that provide chance-constrained optimization models (Rawls and Turnquist, 2011; Hong et al., 2015; Elçi and Noyan, 2018).

The majority of the studies related to post-disaster humanitarian operations assume that the locations of the last mile facilities are known and focus on distribution problems addressing vehicle routing and/or supply allocation decisions. Only a few studies are concerned with last mile network design decisions such as the locations and capacities of the Points of Distribution (PODs) of the relief supplies. Moreover, most of the studies taking into account the decisions related to the locations of the last mile facilities either assume a deterministic setting and/or do not incorporate the concepts of accessibility and equity. Based on these reflections, Noyan et al. (2016) contribute to the literature by introducing a last mile distribution network design problem and presenting a mathematical model that incorporates accessibility and equity while capturing the uncertain aspects of the post-disaster environment. We refer to their study and the references therein for the relevant literature on last mile humanitarian relief logistics and a detailed discussion on the significance of considering the equity and accessibility issues and the inherent uncertainties in the context of last mile relief network design. Kahvecioglu (2014) extends the study of Noyan et al. (2016) by studying a more elaborate integrated last mile network design problem, which relaxes the assumption that there exists a single Local Distribution Center (LDC) with a pre-determined location, and assumes that there already exist some resources located before a disaster occurs and integrates the decisions on the reallocation of pre-stocked relief supplies. At a high level, several aspects differentiate the current work from these previous studies. Noyan et al. (2016) propose a hybrid allocation policy that can balance the trade-off between equity and accessibility and develop a risk-neutral two-stage stochastic programming model with this hybrid supply allocation policy embedded. The current study introduces a new post-disaster relief network design problem and devises a different hybrid supply allocation policy that leverages the chance-constrained framework in focus in order to provide accessible and equitable service to the beneficiaries. Consequently, we move away from the risk-neutral paradigm and construct a novel risk-averse optimization model for post-disaster management following the modeling approach presented in Section 3. We elaborate more on the differences in the problem descriptions and model settings in the next section.

4.2 Problem Description We aim to design a two-echelon system, where the relief supplies arriving at an LDC are sent to PODs in the first echelon, and the relief supplies are delivered from PODs to the beneficiaries in the second echelon. We assume that there is a single LDC with a pre-determined location, and consider a single type of relief item that can be a bundle (standard kit) of critical relief supplies, such as prepackaged food, medical kits, and water.

Last mile relief networks must be set up quickly before the relief organizations can collect accurate information about the post-disaster conditions. This essentially implies that the relief organizations need to make the design decisions before the uncertainties related to the post-disaster conditions are resolved. In line with this viewpoint, Noyan et al. (2016) develop a two-stage model, where the first-stage decisions are for locating the PODs and the second-stage decisions are related to the allocation of supplies to the PODs and the assignments of the demand points to the PODs. Alternatively, we consider the situations where the relief organizations need to make all network design decisions immediately in order to start delivering the relief supplies to the affected areas. To this 
end, we propose a single-stage stochastic programming model, which jointly determines the decisions related to the following: $i$ ) the locations of the PODs, ii) the assignments of the demand points to the PODs, and iii) the distribution of the supplies to the PODs and the demand points.

Following suit with Noyan et al. (2016), we develop an optimization model incorporating the accessibility and equity issues critical to the design of the last mile networks. We follow their approach of characterizing accessibility, defined as the ease of access to the relief supplies. In particular, the accessibility in the first echelon of the last mile network is affected by the physical factors only (e.g., geographical, topographical), while accessibility in the second echelon is affected both by the physical and demographical/socio-economical factors (e.g., age, gender, disability). Noyan et al. (2016) consider an accessibility metric based on the sum of the expected total accessibility of the PODs from the LDC and the expected total accessibility of the PODs from the demand locations. We alternatively design a delivery amount-weighted version of this accessibility metric. Considering more detailed supply distribution decisions (in addition to the decisions on the deliveries from the LDC to the PODs we also determine the amounts of supplies delivered from the PODs to the demand points) allows us to obtain this finer accessibility metric. In order to be consistent with our cost minimization setup, we use the convention that lower accessibility scores indicate higher accessibility and define the accessibility scores as the weighted travel times. These accessibility scores correspond to the reciprocals of those used in Noyan et al. (2016).

We consider two types of equity: equitable accessibility and equitable supply allocation. As in Noyan et al. (2016), we ensure equitable accessibility to the PODs from the demand locations by defining the coverage sets according to a maximum threshold requirement in terms of the accessibility scores associated with the links. However, we take a different approach to modeling equity in supply allocation. Noyan et al. (2016) hybridize two supply allocation policies based on quantitative measures: a strict proportional allocation policy referred to as the PD Policy, which divides the available supply among the PODs in proportion to the total demands assigned to the PODs under each scenario, and the TD policy, which ensures that the shortage amount at each POD does not exceed a specified proportion of the corresponding total demand under each scenario. In particular, their hybrid approach enforces the TD policy and a relaxed version of the PD policy, and penalizes the deviations from the strict PD policy in the objective function in order to distribute the supplies among the PODs in proportion to their total demands as much as possible without compromising from the expected total accessibility. In contrast, in this study, we consider the supply allocations at the demand level instead of the POD level due to the focus on more detailed supply distribution decisions. This property of our model renders the existing TD and PD policies not directly applicable to our setup because the targeted demand levels in our demand satisfaction constraints are the individual demands at each location. These demand values are input parameters while the total demands assigned to the PODs are decision variables implied by the assignment decisions. In our setting, we incorporate both a qualitative and a quantitative measure regarding the stochastic demand satisfaction constraints. As the qualitative measure, we introduce individual chance constraints on satisfying the demands at each location, where the lower bound on the reliability level is set to be equal for each demand location. The quantitative measure is in the form of (23) and closely related to the proportion of unsatisfied demand at each point. Focusing on such a proportional measure is in itself promising in terms of serving the population groups equitably. These approaches lead to a new hybrid supply allocation policy which enforces a set of individual chance constraints on satisfying the demands at each node and penalizes the cost of reliability associated with these demand satisfaction constraints.

4.3 Stochastic Optimization Model We consider a network where each node represents a geographical area (a settlement such as a village and a town) according to the size of the affected region. We denote the set of demand nodes by $I$ and the set of candidate PODs by $J$, where we assume that $J \subseteq I$ without loss of generality. The randomness in the node demands and the accessibility scores associated with the links of the network are represented by a finite set of scenarios. Taking a conservative approach, a POD can cover (serve) 
a demand node only if the worst (largest) accessibility score to the POD from the demand location under any scenario is no larger than a maximum threshold $\bar{\tau}$. Next, we provide the list of the additional input parameters:

$p^{i}$ : probability of scenario $i, i \in S$,

$\xi_{k}$ : the random variable associated with the demand at node $k, k \in I$,

$\xi_{k}^{i}$ : demand at node $k$ under scenario $i$ with the corresponding probability $p_{k}^{i}, k \in I, i \in S ; \xi_{k}^{1} \geq \cdots \geq \xi_{k}^{N}$ holds without loss of generality for all $k \in I$,

$\nu_{0 j}^{i}$ : score for accessibility to candidate POD $j$ from the LDC under scenario $i, j \in J, i \in S$,

$\nu_{k j}^{i}$ : score for accessibility to candidate POD $j$ from demand node $k$ under scenario $i, k \in I, j \in J, i \in S$ with $\nu_{j j}^{i}=0$ for all $j \in J, i \in S$,

$H_{k}=\left\{j \in J \mid \max _{i \in S} \nu_{k j}^{i} \leq \bar{\tau}\right\}$ : set of candidate PODs that can cover demand node $k, k \in I$,

$L_{j}=\left\{k \in I \mid \max _{i \in S} \nu_{k j}^{i} \leq \bar{\tau}\right\}:$ set of demand nodes that can be covered by the candidate POD $j, j \in J$,

$\kappa$ : maximum number of PODs to be opened $(\kappa \leq|J|)$,

$\Theta$ : the total amount of available supplies,

$K_{j}$ : an upper bound on the amount of supplies that can be delivered to POD $j, j \in J$.

In our modeling framework, the network design decisions are made before the uncertainty in the parameters is resolved. We use the notation below for the network design decisions of interest:

- $\gamma_{j}=1$ if a POD is located at node $j \in J$, and $\gamma_{j}=0$ otherwise,

- $\delta_{k j}=1$ if demand node $k \in I$ is served by POD $j \in H_{k}$, and $\delta_{k j}=0$ otherwise,

- $x_{k j}$ : the amount of supplies delivered to demand node $k \in I$ from POD $j \in J$.

Then, the amount of supplies delivered to POD $j$ from the LDC is given by $\sum_{k \in L_{j}} x_{k j}$.

In our modeling approach, we ensure equitable accessibility by serving each demand point by a POD in its coverage set defined based on a common upper bound on the accessibility scores associated with the links. Regarding accessibility, we also design an efficiency-related objective function featuring the total accessibility. We note that the upper bound parameter $\bar{\tau}$ can be used to balance the trade-off between the equity as measured by the worst possible accessibility score and efficiency as quantified by the total accessibility. Moreover, we additionally take into account equity in supply allocation by enforcing a set of individual chance constraints to distribute the relief supplies to the demand points in such a way that the probability of satisfying the demand at each node is at least equal to a common a lower bound $(1-\bar{\epsilon})$. In addition to this qualitative measure on the level of demand satisfaction, we incorporate a quantitative measure - based on the deviations from the desired levels of delivery - into the objective function to control for the demand shortage amounts. Following the modeling approach proposed in Section 3.2, we set the desired level of delivery for node $k$ as $\operatorname{VaR}_{\left(1-\epsilon_{k}\right)}\left(\xi_{k}\right)$, $k \in I$, and calculate the proportion of unsatisfied demand (PUD) based on these lower threshold VaR values. This modeling approach based on the variable reliability levels allows us to balance the trade-off between the total accessibility and the cost of reliability associated with the demand satisfaction in the objective function. We next present the chance-constrained optimization model developed for our stochastic relief network design problem:

(CCSRND)

$$
\begin{aligned}
\min _{\boldsymbol{\gamma}, \boldsymbol{\delta}, \mathbf{x}, \boldsymbol{\epsilon}} & \sum_{i \in S} \sum_{j \in J} p^{i} \nu_{0 j}^{i} \sum_{k \in L_{j}} x_{k j}+\sum_{i \in S} \sum_{k \in I} \sum_{j \in H_{k}} p^{i} \nu_{k j}^{i} x_{k j} \\
& +\sum_{k \in I} \sum_{i \in\left[i_{k}^{*}-1\right]} p_{k}^{i}\left(\frac{a}{\xi_{k}^{i}}\left[\xi_{k}^{i}-\operatorname{VaR}_{\left(1-\epsilon_{k}\right)}\left(\xi_{k}\right)\right]_{+}\right) \\
\text {s.t. } & \sum_{j \in J} \gamma_{j} \leq \kappa,
\end{aligned}
$$




$$
\begin{array}{lr}
\sum_{k \in L_{j}} x_{k j} \leq K_{j} \gamma_{j}, & \forall j \in J \\
\sum_{j \in J} \sum_{k \in L_{j}} x_{k j}=\Theta, & \\
\sum_{j \in H_{k}} x_{k j} \leq \max _{i \in S} \xi_{k}^{i}, & \forall k \in I \\
\sum_{j \in H_{k}} \delta_{k j}=1, & \forall k \in I \\
\delta_{k j} \leq \gamma_{j}, & \forall k \in I, j \in H_{k} \\
\delta_{j j} \geq \gamma_{j}, & \forall j \in J \\
x_{k j} \leq \delta_{k j} U_{k j}, & \forall k \in I, j \in H_{k} \\
P\left(\sum_{j \in H_{k}} x_{k j} \geq \xi_{k}\right) \geq 1-\epsilon_{k}, & \forall k \in I \\
0 \leq \epsilon_{k} \leq \bar{\epsilon}, & \\
\gamma_{j} \in\{0,1\}, & \forall k \in I \\
\delta_{k j} \in\{0,1\}, & \forall j \in J \\
x_{k j} \geq 0, & \forall k \in I, j \in H_{k}
\end{array}
$$

The objective function (26a) minimizes the expected total delivery amount-weighted accessibility score (ETWA) and the expected cost of the risk tolerances $\epsilon_{k}, k \in I$, where the expected values are estimated by sample averaging. The second cost term is based on the VaR-based PUD values referred to as V-PUD. Thus, the expected cost of the reliability levels is specified as a linear function of the expected total V-PUD. Considering the V-PUD values and assigning a common penalty coefficient $a$ to all demand points are consistent with the intent of fair supply allocation. Constraint (26b) ensures that the number of established PODs is not larger than the specified limit $\kappa$. Constraints (26c) play a dual role by imposing a maximum capacity limit on the amount of supplies delivered to each open POD and mandating that there is a POD located at node $j$ if there is any delivery to that POD. According to the constraints (26d) and (26e), all available supplies are distributed in the network while the total supply amount allocated to a demand node does not exceed its largest possible demand realization. Here, the underlying assumption is that $\Theta \leq \sum_{k \in I} \max _{i \in S} \xi_{k}^{i}$. This relation may not hold only in rare and uninteresting cases when the relief supplies in the last mile network are ample compared to the total demand. Constraints (26f) and (26g) guarantee that each demand node is assigned to a single open POD - a policy known as single sourcing and implemented commonly in practice. A demand node with an open POD should naturally be served by this POD - as is also favored by the objective coefficients $\nu_{j j}^{i}=0, j \in J, i \in S$. However, under extremely restrictive capacity limits, the optimal solution may not obey this rule. To avoid such peculiar solutions, we embed this rule explicitly via constraints (26h). Constraints (26i) set the delivery amounts to the demand nodes from their respective assigned PODs. The delivery amount to node $k$ is naturally bounded from above by the capacity of its corresponding POD and the maximum possible demand realization at node $k$; that is, $U_{k j}:=\min \left\{K_{j}, \max _{i \in S} \xi_{k}^{i}\right\}$ for all $k \in I$ and $j \in H_{k}$. The constraints $(26 \mathrm{j})$ and $(26 \mathrm{k})$ collectively prescribe that the probability of satisfying demand at each demand node is at least $1-\epsilon_{k} \geq 1-\bar{\epsilon}$. The rest of the constraints enforce the non-negativity and binary restrictions.

Drawing upon the tools of Sections 2 and 3 in order to reformulate the individual chance constraints $(26 \mathrm{j})$ and the cost of reliability in the objective function (26a) yields the MIP reformulation of (CCSRND) below:

$$
\min _{\boldsymbol{\gamma}, \boldsymbol{\delta}, \mathbf{x}, \mathbf{z}, \boldsymbol{\epsilon}, \boldsymbol{\beta}} \sum_{i \in S} \sum_{j \in J} p^{i} \nu_{0 j}^{i} \sum_{k \in L_{j}} x_{k j}+\sum_{i \in S} \sum_{k \in I} \sum_{j \in H_{k}} p^{i} \nu_{k j}^{i} x_{k j}+\sum_{k \in I} \sum_{i \in\left[i_{k}^{*}-1\right]} p_{k}^{i}\left(\frac{a}{\xi_{k}^{i}} w_{k i}\right)
$$




$$
\begin{aligned}
\text { subject to } & (26 \mathrm{~b})-(26 \mathrm{i}),(26 \mathrm{k})-(26 \mathrm{n}), \\
& \sum_{j \in H_{k}} x_{k j} \geq z_{k}, \\
& (15 \mathrm{c})-(15 \mathrm{e}),(15 \mathrm{~h}),(22 \mathrm{c})-(22 \mathrm{e}),(24)-(25) .
\end{aligned}
$$

The formulation (27) is precisely in the form of (SMICC - VRTQA - $\boldsymbol{\beta})$. The one to one relation is easily observed by noting that $[m]$ and $[N]$ in (SMICC - VRTQA - $\beta$ ) correspond to $I$ and $S$ in (CCSRND), respectively.

Remark 4.1 As discussed in Section 2, additional constraints on the variable risk tolerance decisions may also be included in (27). For instance, it would be natural to bound the summation of the risk tolerances as in (19c), where the generic set $[\mathrm{m}]$ is replaced by $I$. It is easy to show that adding (19c) to the formulation (27) guarantees that the joint chance constraint $\mathbb{P}\left(\sum_{j \in H_{k}} x_{k j} \geq \xi_{k}, \quad \forall k \in I\right) \geq 1-\Gamma$ holds for any of its feasible solutions. If the number of individual chance constraints is large, such a joint chance constraint can be very demanding for $\Gamma<1$. In practical applications, setting $\Gamma \geq 1$ in (19c) may therefore be preferable - ignoring the connection with the joint chance constraint mentioned above. We also take this path in Section 5.

For the post-disaster application in focus, the proposed modeling approach grants a flexibility to balance the trade-off between the accessibility captured by ETWA and the level of demand satisfaction. One can impact this trade-off by changing the values of the parameters $a$ and $\bar{\epsilon}$. We intend to provide more insights about the model by presenting numerical results on a case study in Section 5.3.

5. Computational Study In the computational study, we fulfill the objectives and claims set forth in the previous sections. This is accomplished in two parts. In the first part, we demonstrate the computational effectiveness of the proposed MIP formulations for the two types of chance-constrained optimization models of interest (MICC - VRT) and (MICC - VRTQ). For (MICC - VRT), we provide clear evidence that the new formulations (SMICC - VRT $-\boldsymbol{\beta}$ ) and (SMICC - VRT $-\mathbf{y}$ ) outperform the formulation (MICC - VRT - DEF) due to Shen (2014) by a significant margin. The impact of the alternate VaR representations in Lemma 2.2 on the computational performance is investigated in the context of (MICC - VRTQ) by benchmarking (SMICC - VRTQ - $\boldsymbol{\beta}$ ) against its counterpart with the alternate VaR representation (14). The runs in this part also reveal that the formulations based on the $\beta$ - and $y$-variables may indeed exhibit disparate performances. In the second part, we illustrate the application of the proposed stochastic relief network design model (CCSRND) on a case study developed based on the real-world data from the 2011 Van earthquake in Turkey. The takeaway from the numerical results here is that solving large practical instances to optimality is within the reach of the proposed formulations.

All runs are executed on a personal computer with a $3.2 \mathrm{GHz}$ Intel ${ }^{\circledR}$ Core $^{\mathrm{TM}}$ i7 960 processor and $24 \mathrm{~GB}$ of memory running on Windows 7. All MIP formulations are implemented and solved in C++ using the Concert Technology component library of IBM ${ }^{\circledR}$ ILOG ${ }^{\circledR}$ CPLEX ${ }^{\circledR}$ 12.6. CPLEX is invoked with a time limit of 3600 seconds and its default set of options and parameters. All reported times are elapsed times in seconds. If optimality is not proven within the allotted time, we record both the best lower bound LB on the optimal objective value retrieved from CPLEX and the objective function value associated with the incumbent at termination - denoted by UB. Then, we calculate an upper bound on the relative optimality gap of the incumbent as $\mathrm{ROG}=(U B-L B) / L B$.

As underlined in Section 1, one of the promises of this paper is to empower practitioners to solve large-scale individual chance-constrained optimization problems by demonstrating good modeling practices put forward by recent methodological progress. In other words, the emphasis on solving all MIP formulations by an off-the-shelf solvers is deliberate. If more speed is desired, then the strengthened star inequalities - see Lemma 2.1-(v) may be handled differently in a more advanced implementation of the formulations with the $\beta$-variables. In 
the current state, we add a single strengthened star inequality for each individual chance constraint, sufficient to yield a valid reformulation of the original problem with big- $M$ constraints. Alternatively, we can develop an iterative solution method based on the entire set of strengthened star inequalities, which is exponential in size (Theorem 2, Luedtke et al., 2010). Therefore, in this case, it is natural to follow a delayed cut generation approach, which avoids explicitly constructing exponentially many constraints in advance. A corresponding branch-and-cut implementation would only initialize with a small subset of the strengthened star inequalities and then add missing ones as required by solving an appropriate separation subproblem in the search tree. Such an implementation would potentially result in quicker solution times.

5.1 Generation of the Problem Instances We performed our computational tests on two types of problems: a probabilistic version of the classical transportation problem with random demand (Luedtke et al., 2010) and the stochastic last mile relief network design problem with random demand and accessibility scores presented in Section 4.

For the first problem type, we use the data sets of Luedtke et al. (2010) with "general probabilities," as labeled by the authors. In these instances, the scenarios may have different probabilities. The authors randomly generated instances with the number of suppliers fixed at 40 and varying numbers of customers $(n=100,200)$ and scenarios $(|S|=1000,2000$ for instances with 100 customers and $|S|=2000,3000$ for instances with 200 customers). Further details are available in the original paper. We refer to these problem instances as "transportation (TR) instances" and note that for some analyses we also created smaller TR instances with 100 customers by deleting scenarios from the original instances with $n=100$ and $|S|=1000$.

For the second problem type, we focus on the Van earthquake relief case study developed by Noyan et al. (2016). We used the readily available instances and also generated additional larger instances by following their data generation scheme. The network in this case study contains 94 demand points, each representing one settlement in the main district of Van. The authors also construct networks with 30 and 60 nodes by clustering the original set of settlements via a $P$-median model that minimizes the demand-weighted travel times. We refer to these problem instances as "relief network design (RND) instances". For the details of the data collection and scenario generation procedures, we refer the reader to Noyan et al. (2016) and the associated online supplement.

Here, we briefly recall some parameters from Noyan et al. (2016) and comment on the parameters whose values are specified differently from those in the original paper. The amount of available supplies $\Theta$ is set as $110 \%$ of the expected total demand. The parameter $\kappa$ is selected as 6,9 , and 12 for the networks with 30,60 , and 94 nodes, respectively. The tightness of the capacity upper bounds are varied to illustrate their impact. In particular, we set $K_{j}=c \hat{\xi_{j}}$, where $\hat{\xi}_{j}$ denotes the estimated base value of the demand at node $j \in J$, and $c$ is selected from the range $[1.75,8]$. Another influential parameter is the upper bound $\bar{\tau}$ on the accessibility scores, which determines the size of the coverage sets, and is calculated as $\bar{\tau}=1 / \tau$. To avoid overly small coverage sets, $\tau$ is selected from the range $[0.01,0.15]$.

5.2 Computational Performance We summarize the common elements in the design of the numerical experiments in this section before proceeding with the performance analysis. First, recall that in general two types of constraints are imposed on the variable risk tolerances $\epsilon_{k}, k \in[m]$ - the constraints (9d) and the simple upper bound constraints (9e). Unless otherwise stated, here all formulations only include the latter, and the parameter $\bar{\epsilon}$ - the upper bound on the risk tolerances - is set as 0.3 for both types of problem instances. In some analyses with the TR instances, the risk budget constraint (19c) is incorporated in addition to the constraints (9e). In these experiments, the set of constraints (9d) takes the form of (19c), and $\Gamma$ is set as 3 and 6 for the instances with 100 and 200 demand nodes, respectively. Second, for each combination of the parameters in the tables in this section, three instances were generated by following the instructions in Section 5.1. All results presented in the tables are therefore averaged over three instances. Third, unless otherwise stated, CPLEX is allowed to use up to four parallel threads as specified by its Threads parameter. Finally, note 
that the common string "MICC" is dropped from the formulation names in the column headers of the tables for ease of presentation.

5.2.1 Evaluation of the Alternate Formulations of (MICC - VRT) In this section, we assess how our proposed formulations (SMICC - VRT $-\boldsymbol{\beta}$ ) and (SMICC - VRT - y) fare against (MICC - VRT - DEF) presented by Shen (2014). Here, we deliberately stick with the formulation $($ SMICC - VRT - $\boldsymbol{\beta})$ for the $\beta$-variables because the VaR representation embedded in this formulation and the VaR representation employed by Shen (2014) in (MICC - VRT - DEF) both rely on Lemma 2.1-(iii). Thus, any difference in performance can mainly be attributed to how the quantiles are captured in the reformulation of the chance constraints based on (4).

Time [ROG (\%)]

\begin{tabular}{|c|c|c|c|c|c|c|c|}
\hline$n$ & $|S|$ & $\begin{array}{r}\text { Budget } \\
(\text { VRT - DEF })\end{array}$ & $\begin{array}{c}\text { constraint }(19 \mathrm{c}) \\
(\mathbf{S}-\mathbf{V R T}-\boldsymbol{\beta})\end{array}$ & $\begin{array}{l}\text { omitted } \\
\qquad(\mathbf{S}-\text { VRT }-\mathbf{y})\end{array}$ & $\begin{array}{r}\text { Budget } \\
(\text { VRT }- \text { DEF })\end{array}$ & $\begin{array}{r}\text { constraint }(19 \mathrm{c}) \\
(\mathbf{S}-\mathbf{V R T}-\boldsymbol{\beta})\end{array}$ & $\begin{array}{l}\text { included } \\
\qquad(\mathbf{S}-\mathbf{V R T}-\mathbf{y})\end{array}$ \\
\hline \multirow[t]{6}{*}{100} & 50 & $\dagger^{\dagger \dagger}[53.4]$ & 0.1 & 0.1 & †††[38.8] & 0.1 & 0.1 \\
\hline & 100 & $\dagger^{\dagger \dagger}[67.7]$ & 0.2 & 0.2 & $\dagger+\dagger[59.0]$ & 0.2 & 0.2 \\
\hline & 200 & †† [77.7] & 0.2 & 0.3 & $\dagger^{\dagger \dagger}[76.0]$ & 0.2 & 0.2 \\
\hline & 500 & †† [86.3] & 0.4 & 0.5 & †††[86.3] & 0.4 & 0.4 \\
\hline & 1000 & $\dagger^{\dagger \dagger}[90.6]$ & 0.9 & 0.9 & †††[90.6] & 0.8 & 0.8 \\
\hline & 2000 & †† [93.5] & 1.9 & 1.7 & $\dagger \dagger \dagger[93.5]$ & 2.0 & 1.5 \\
\hline \multirow[t]{3}{*}{200} & 2000 & ${ }^{\dagger \dagger \dagger}[94.6]$ & 4.4 & 3.3 & $\dagger^{\dagger \dagger}[94.5]$ & 4.9 & 3.0 \\
\hline & 3000 & $\dagger^{\dagger \dagger[95.9]}$ & 8.7 & 5.2 & $\dagger+\dagger[95.9]$ & 9.0 & 4.4 \\
\hline & Avg & $3600[82.5]$ & 2.1 & 1.5 & 3600 [79.3] & 2.2 & 1.3 \\
\hline
\end{tabular}

Table 2: Computational performance of the alternate MIPs for (MICC - VRT) on the TR instances.

The computational effectiveness of all three formulations is assessed on the two benchmark sets specified in Section 5.1. The results for the TR and RND instances are depicted in Tables 2 and 3, respectively. In these tables, we report the average solution times and ROG values. The ROG information is skipped altogether if all three instances associated with a cell terminate with an optimal solution. The values of the parameter $a$ are specified as 1,000,000 and 100,000 for the TR and RND instances, respectively. For the RND instances, the values of the parameters $(\tau, c)$ are a major determinant of instance difficulty as demonstrated in the subsequent sections. Here, $(\tau, c)$ are specified as $(0.01,2.00),(0.05,2.25)$, and $(0.09,2.75)$ for the instances with $|I|=30,60$, and 94 nodes, respectively.

The figures in Tables 2 and 3 reveal that (MICC - VRT - DEF) is outperformed drastically by our MIP formulations (SMICC - VRT - $\boldsymbol{\beta}$ ) and (SMICC - VRT - y), which generally perform on a par. No significant pattern is evident from a comparison of their solution times. (MICC - VRT - DEF) fails to provide a single optimal solution within the time limit except for the RND instances with $|I|=94$ and $|S|=50$. In stark contrast, all average solution times associated with $(\mathbf{S M I C C}-$ VRT $-\boldsymbol{\beta})$ and $(\mathbf{S M I C C}-\mathbf{V R T}-\mathbf{y})$ are below 10 and 90 seconds in Tables 2 and 3, respectively, except for the large RND instances with $|I|=94$ and $|S|=200$. For illustrative purposes, in Table 2 we also investigate the sensitivity of the formulations to the presence of (19c). We do not detect any effect and conclude that the results in Table 2 are robust with respect to this type of coupling risk budget constraint.

5.2.2 Evaluation of the Alternate Formulations of (MICC - VRTQ) In this section, we rate the performance of the formulations available for (MICC - VRTQ). For the RND instances, the cost of reliability $h_{k}\left(z_{k}\right)$ in the objective function is specified as in (23), where the coefficients $a_{k i}$ are defined as $\frac{a}{\xi_{k}^{i}}$. For diversity, 
Time $[$ ROG (\%)]

$|I|=30 \quad|I|=60 \quad|I|=94$

$|S||(\mathbf{V R T}-\mathbf{D E F})(\mathbf{S}-\mathbf{V R T}-\boldsymbol{\beta})(\mathbf{S}-\mathrm{VRT}-\mathbf{y})|(\mathbf{V R T}-\mathrm{DEF})(\mathbf{S}-\mathbf{V R T}-\boldsymbol{\beta})(\mathbf{S}-\mathrm{VRT}-\mathbf{y}) \mid(\mathbf{V R T}-\mathrm{DEF})(\mathbf{S}-\mathrm{VRT}-\boldsymbol{\beta})(\mathbf{S}-\mathbf{V R T}-\mathbf{y})$

\begin{tabular}{r|cll|lll|lccc}
\hline 50 & $\dagger \dagger \dagger[36.8]$ & 0.6 & 0.6 & $\dagger \dagger[50.2]$ & 40.7 & 47.8 & 164.4 & 7.7 & 8.2 \\
100 & $\dagger \dagger \star[68.2]$ & 0.8 & 1.2 & $\dagger \dagger \dagger[36.7]$ & 48.7 & 39.0 & $\dagger \dagger[26.7]$ & 38.4 & 81.2 \\
200 & $\dagger \dagger \star[70.7]$ & 1.1 & 1.2 & $\dagger \dagger \dagger[39.5]$ & 6.8 & 5.7 & $\dagger \dagger *[48.8]$ & 596.8 & 1040.7 \\
500 & $\dagger \dagger \dagger[62.6]$ & 1.7 & 1.5 & $\dagger \dagger \dagger[49.8]$ & 15.9 & 15.0 & $\dagger \star *[78.3]$ & 9.8 & 9.4 \\
\hline Avg & $3600[59.6]$ & 1.0 & 1.1 & $3600[44.1]$ & 28.0 & 26.9 & $2742.5[38.4]$ & 163.2 & 284.9 \\
\hline
\end{tabular}

${ }^{\dagger}$ : Each dagger sign indicates one instance hitting the time limit with an integer feasible solution.

${ }^{\star}$ : Each asterisk sign indicates one instance hitting the time limit with no integer feasible solution. In this case, we assume that the associated ROG value is $100 \%$.

Table 3: Computational performance of the alternate MIPs for (MICC - VRT) on the RND instances.

we employ the term $h_{k}\left(z_{k}\right)=-a_{k} z_{k}=-a z_{k}$ for the TR instances. We observe that the VaR values can be significantly larger than the values of the reliability levels. Therefore, we determine the values of the parameter $a$ by running some preliminary tests in order to ensure that the values of the risk tolerances in the optimal solutions are not necessarily always at the end points of the range $[0, \bar{\epsilon}]$. For the analyses with the TR and RND instances, the parameter $a$ assumes the values 50 and 1,000,000, respectively.

Time

\begin{tabular}{cc|cc}
\cline { 3 - 4 }$n$ & $|S|$ & VaR Rep (13) & VaR Rep (14) \\
\hline 100 & 2000 & 13.9 & 78.2 \\
200 & 2000 & 139.2 & 357.3 \\
200 & 3000 & 199.3 & 753.1 \\
\hline \multicolumn{2}{r|}{ Avg } & 117.5 & 396.2 \\
\hline
\end{tabular}

Table 4: Computational performance of the alternate VaR representations on the TR instances.

\begin{tabular}{c|cc|cc|ccc}
\multicolumn{7}{c}{ Time } \\
\cline { 2 - 7 }$|S|$ & VaR Rep (13) & VaR Rep & $(14)$ & VaR Rep (13) & VaR Rep (14) & VaR Rep (13) & VaR Rep (14) \\
\hline 1000 & 18.2 & 18.7 & 101.1 & 81.0 & 102.9 & 108.8 \\
2000 & 24.6 & 31.6 & 146.3 & 127.9 & 113.7 & 106.1 \\
3000 & 38.9 & 49.5 & 133.4 & 221.2 & 187.3 & 155.2 \\
5000 & 58.1 & 60.6 & 217.1 & 218.8 & 311.2 & 296.9 \\
\hline Avg & 35.0 & 40.1 & 149.5 & 162.2 & 178.8 & 166.8 \\
\hline$\tau$
\end{tabular}

Table 5: Computational performance of the alternate VaR representations on the RND instances.

Our first goal in this section is to expose the computational differences between the alternate VaR representations listed in Lemma 2.2 for our formulations based on the $\beta$-variables and stick to one of these for the rest of the computational study. To this end, we assess the performance of (SMICC - VRTQ - $\boldsymbol{\beta}$ ) against that of its counterpart with the alternate VaR representation (14). The largest TR and RND instances are all solved to optimality with both representations, and the average solution times are presented in Tables 4 and 5 , respectively. In this analysis, the risk budget constraint (19c) is included for the runs with the TR instances. As pointed out following the proof of Lemma 2.2, (14) is a stronger representation compared to (13) from a theoretical viewpoint. However, it appears that this fact does not necessarily translate into computational gains, and 
the results in Tables 4-5 refute the expectation that the representation (14) should consistently lead to smaller solution times. In complete contrast, the average solution times associated with (13) are substantially smaller in comparison to those obtained with (14) on the TR instances. For the RND instances, the picture is mixed. The presentation (13) has a slight edge over (14) on the smaller instances with $I=|30|$, while the reverse is true for the larger instances with $I=|94|$. No definitive pattern emerges for the remaining instances with $I=|60|$. We decide that the presentation (13) delivers a better performance overall, and going forward the VaR representation (13) is incorporated into the formulations (SMICC - VRTQ - $\boldsymbol{\beta}$ ) and (SMICC - VRTQA - $\boldsymbol{\beta}$ ) for the TR and RND instances, respectively.

Time

\begin{tabular}{|c|c|c|c|c|c|}
\hline \multirow[b]{2}{*}{$n$} & \multirow[b]{2}{*}{$|S|$} & \multicolumn{2}{|c|}{ Budget constraint (19c) omitted } & \multicolumn{2}{|c|}{ Budget constraint (19c) included } \\
\hline & & $(\mathbf{S}-\mathbf{V R T Q}-\mathbf{y})$ & $(\mathbf{S}-$ VRTQ $-\boldsymbol{\beta})$ & $(\mathbf{S}-\mathbf{V R T Q}-\mathbf{y})$ & $(\mathbf{S}-$ VRTQ $-\boldsymbol{\beta})$ \\
\hline \multirow[t]{4}{*}{100} & 200 & 0.5 & 0.7 & 0.8 & 1.5 \\
\hline & 500 & 1.0 & 1.2 & 1.6 & 6.0 \\
\hline & 1000 & 1.6 & 0.9 & 3.1 & 26.4 \\
\hline & 2000 & 2.5 & 1.5 & 5.2 & 13.9 \\
\hline \multirow[t]{3}{*}{200} & 2000 & 5.1 & 5.7 & 20.7 & 139.2 \\
\hline & 3000 & 7.6 & 9.1 & 14.5 & 199.3 \\
\hline & Avg & 3.1 & 3.2 & 7.7 & 64.4 \\
\hline
\end{tabular}

Table 6: Computational performance of the alternate MIPs for (MICC - VRTQ) on the TR instances.

Next, we turn our attention to whether the formulations based on the $\beta$ - and $y$-variables act similarly as they do in the previous section. The results for $(\mathbf{S M I C C}-\mathbf{V R T Q}-\mathbf{y})$ and $(\mathbf{S M I C C}-\mathbf{V R T Q}-\boldsymbol{\beta})$ on the TR instances are provided in Table 6, where we distinguish between the presence and absence of the risk budget constraint (19c). Both formulations terminate with an optimal solution within the time limit for all instances, but there exists a marked difference in comparison to the results in Section 5.2 .1 in one important aspect. The two formulations are virtually indistinguishable in the absence of the budget constraint. However, the detrimental effect of the inclusion of $(19 \mathrm{c})$ on $(\mathbf{S M I C C}-\mathbf{V R T Q}-\boldsymbol{\beta})$ is much more substantial than on (SMICC - VRTQ - y). For the RND instances, (SMICC - VRTQA - y) is benchmarked against (SMICC - VRTQA - $\boldsymbol{\beta})$ - recall that the latter boils down to the formulation (CCSRND) presented in Section 4.3. Table 7 depicts the outcome, where the common string "SMICC - VRT" is dropped from the formulation names for brevity. The results in Table 7 also control for the values of the combination of the parameters $\tau$ and $c$, which play a pivotal role in the design of the relief networks. Demanding values of these parameters might lead to infeasible solutions - see Section 5.3, and they also affect the solution times, as is evident from Table 7. In any case, all instances are solved to optimality with both formulations; however, there is a shift in performance with increasing problem size. For $|S|=500,1000,($ SMICC - VRTQA - y) is somewhat better; however, (SMICC - VRTQA - $\boldsymbol{\beta})$ starts to dominate for larger number of scenarios.

One critical insight that needs to come across out of this computational study is that it is essential to experiment with the different formulations and VaR representations laid out in this paper. The performance may very much be dependent on the problem and instance settings as clearly illustrated in this section.

5.3 Analysis of (CCSRND) In this section, we first demonstrate the impact of the input parameters $c$, $\tau, a$, and $\bar{\epsilon}$ on the trade-off between the accessibility and the level of demand satisfaction. This is followed by a comparison of the solutions attained by (CCSRND) against those retrieved from a set of benchmark models with the end goal of highlighting the value of the proposed hybrid supply allocation policy. All analyses in this section are performed on the same base instance with $|I|=30,|S|=50$.

The parameters $c$ and $\tau$ respectively determine the level of restrictiveness of the capacity limits and the 


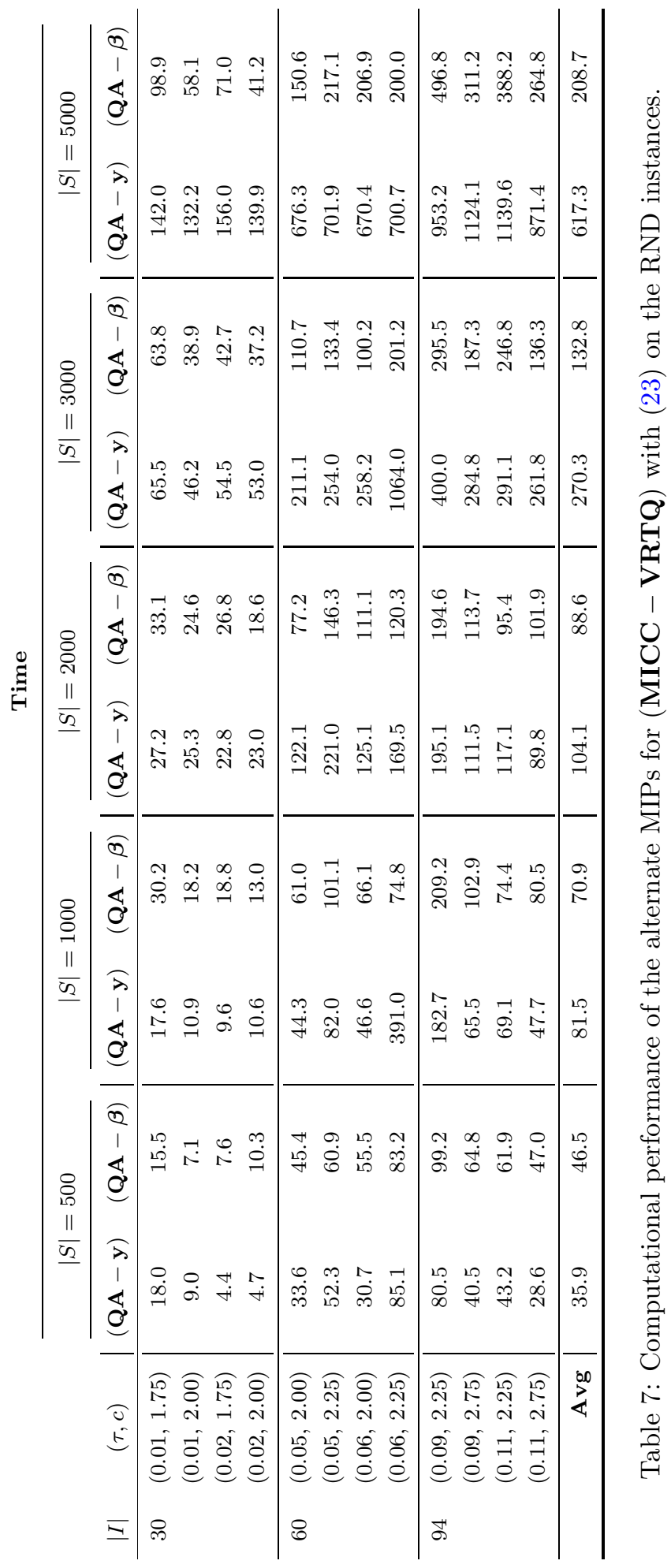




\begin{tabular}{|c|c|c|c|c|c|c|c|c|c|}
\hline \multirow[b]{2}{*}{$c$} & \multirow[b]{2}{*}{$a$} & \multicolumn{2}{|c|}{$\tau=0.01$} & \multicolumn{2}{|c|}{$\tau=0.02$} & \multicolumn{2}{|c|}{$\tau=0.03$} & \multicolumn{2}{|c|}{$\tau=0.04$} \\
\hline & & ETWA & ETV-PUD & ETWA & ETV-PUD & ETWA & ETV-PUD & ETWA & ETV-PUD \\
\hline \multirow{5}{*}{2} & $0 \times 10^{5}$ & 716,460 & 0.4913 & 716,460 & 0.4913 & - & - & - & - \\
\hline & $1 \times 10^{5}$ & 726,165 & 0.2437 & 726,419 & 0.2408 & - & - & - & - \\
\hline & $5 \times 10^{5}$ & 765,978 & 0.0842 & 765,853 & 0.0844 & - & - & - & - \\
\hline & $10 \times 10^{5}$ & 777,922 & 0.0658 & 777,922 & 0.0658 & - & - & - & - \\
\hline & $50 \times 10^{5}$ & 875,154 & 0.0165 & 945,378 & 0.0116 & - & - & - & - \\
\hline \multirow{5}{*}{3} & $0 \times 10^{5}$ & 616,128 & 0.4913 & 616,128 & 0.4913 & 616,128 & 0.4913 & - & - \\
\hline & $1 \times 10^{5}$ & 630,467 & 0.1557 & 630,461 & 0.1558 & 630,404 & 0.1564 & - & - \\
\hline & $5 \times 10^{5}$ & 661,667 & 0.0216 & 661,673 & 0.0216 & 661,650 & 0.0217 & - & - \\
\hline & $10 \times 10^{5}$ & 665,034 & 0.0165 & 665,424 & 0.0161 & 665,001 & 0.0166 & - & - \\
\hline & $50 \times 10^{5}$ & 668,480 & 0.0146 & 668,480 & 0.0146 & 668,480 & 0.0146 & - & - \\
\hline \multirow{5}{*}{4} & $0 \times 10^{5}$ & 612,163 & 0.4913 & 612,163 & 0.4913 & 612,163 & 0.4913 & - & - \\
\hline & $1 \times 10^{5}$ & 625,590 & 0.1554 & 625,590 & 0.1554 & 625,590 & 0.1554 & - & - \\
\hline & $5 \times 10^{5}$ & 652,204 & 0.0208 & 652,412 & 0.0204 & 652,402 & 0.0205 & - & - \\
\hline & $10 \times 10^{5}$ & 657,747 & 0.0130 & 657,335 & 0.0134 & 657,747 & 0.0130 & - & - \\
\hline & $50 \times 10^{5}$ & 660,239 & 0.0116 & 660,239 & 0.0116 & 660,239 & 0.0116 & - & - \\
\hline \multirow{5}{*}{8} & $0 \times 10^{5}$ & 581,449 & 0.4913 & 581,449 & 0.4913 & 581,449 & 0.4913 & 592,197 & 0.4913 \\
\hline & $1 \times 10^{5}$ & 591,662 & 0.1944 & 591,739 & 0.1937 & 591,464 & 0.1964 & 604,498 & 0.1603 \\
\hline & $5 \times 10^{5}$ & 627,514 & 0.0235 & 627,514 & 0.0235 & 627,514 & 0.0235 & 634,141 & 0.0214 \\
\hline & $10 \times 10^{5}$ & 631,732 & 0.0165 & 631,746 & 0.0165 & 631,746 & 0.0164 & 640,656 & 0.0124 \\
\hline & $50 \times 10^{5}$ & 642,051 & 0.0116 & 642,051 & 0.0116 & 642,051 & 0.0116 & 642,051 & 0.0116 \\
\hline
\end{tabular}

Table 8: Values of the objective function terms at optimality for varying parameter settings $(\bar{\epsilon}=0.3)$.

coverage sets while the parameters $a$ and $\bar{\epsilon}$ play critical roles in controlling the trade-off between accessibility and the cost of reliability associated with demand satisfaction. The effects of these parameters on the two conflicting objective function criteria ETWA and the expected total V-PUD - denoted by ETV-PUD - are highlighted in Table 8, where we specifically explore the interactions among $c, \tau$, and $a$ by keeping $\bar{\epsilon}$ fixed at 0.30. Larger values of $c$ imply less restrictive capacity limits and clearly lead to reductions in the optimal objective function value ETWA $+a$ ETV-PUD. These improvements generally translate to reductions in both of the objective function criteria; however, for a number of cases when $c$ is increased from 4 to 8 , the performance degrades with respect to ETV-PUD. We also observe that the deterioration in ETWA as $a$ is increased is more pronounced for $c=2$ in comparison to the higher values of $c$. Another clear insight from Table 8 is that smaller values of $c$ and larger values of $\tau$ can result in infeasibilities. However, when the value of $\tau$ is not large enough to cause infeasibilities, its effect appears to be marginal. To investigate the impact of the parameter $\bar{\epsilon}$, we analyze the attained reliability levels at each demand point under varying values of $\bar{\epsilon}$ and $a$. The values of $c$ and $\tau$ are set as 2 and 0.01 , respectively. In Table 9, the demand nodes are classified into six categories depending on their attained reliability levels / risk tolerances. To clarify, those demand nodes with a minimum attained risk tolerance of $0.95 * 0.20=0.19$ or $0.95 * 0.50=0.475$ are assigned to the row labeled as " $(95 \%-100 \%) \vec{\epsilon}$ " if $\bar{\epsilon}=0.20$ or $\bar{\epsilon}=0.50$, respectively. On the one hand, small values of $\bar{\epsilon}$ already appear to be very effective in ensuring lower costs of reliability. Therefore, the risk tolerance decisions are less sensitive to the changes in the value of $a$ if $\bar{\epsilon}=0.2$. For $\bar{\epsilon}=0.35,0.50$ on the other hand, the expected trend is clearly present - as $a$ assumes larger values the number of demand nodes in the groups with lower risk tolerances increases. In any case, the optimization fully exploits the flexibility in setting the risk tolerances, and the attained values are distributed over the allowable range $[0, \bar{\epsilon}]$.

We next assess the value of the proposed hybrid policy in terms of equity in supply allocation. Recall that this policy features both quantitative and qualitative measures regarding the demand satisfaction, and the 


\begin{tabular}{|c|c|c|c|c|c|c|c|c|c|c|c|c|}
\hline & \multicolumn{12}{|c|}{ \# of demand nodes } \\
\hline & \multicolumn{4}{|c|}{$\bar{\epsilon}=0.20$} & \multicolumn{4}{|c|}{$\bar{\epsilon}=0.35$} & \multicolumn{4}{|c|}{$\bar{\epsilon}=0.50$} \\
\hline & \multicolumn{4}{|c|}{$a\left(\times 10^{5}\right)$} & \multicolumn{4}{|c|}{$a\left(\times 10^{5}\right)$} & \multicolumn{4}{|c|}{$a\left(\times 10^{5}\right)$} \\
\hline & 1 & 2.5 & 5 & 10 & 1 & 2.5 & 5 & 10 & 1 & 2.5 & 5 & 10 \\
\hline$(0 \%-5 \%) \bar{\epsilon}$ & 0 & 0 & 0 & 0 & 0 & 0 & 2 & 15 & 1 & 5 & 9 & 20 \\
\hline$(5 \%-25 \%) \bar{\epsilon}$ & 3 & 3 & 3 & 4 & 7 & 13 & 19 & 10 & 9 & 13 & 15 & 5 \\
\hline$(25 \%-50 \%) \bar{\epsilon}$ & 4 & 3 & 5 & 4 & 7 & 6 & 4 & 0 & 10 & 7 & 4 & 3 \\
\hline$(50 \%-75 \%) \bar{\epsilon}$ & 4 & 8 & 8 & 7 & 7 & 7 & 3 & 3 & 3 & 3 & 2 & 2 \\
\hline$(75 \%-95 \%) \bar{\epsilon}$ & 5 & 5 & 4 & 5 & 2 & 4 & 2 & 2 & 2 & 1 & 0 & 0 \\
\hline$(95 \%-100 \%) \bar{\epsilon}$ & 14 & 11 & 10 & 10 & 7 & 0 & 0 & 0 & 5 & 1 & 0 & 0 \\
\hline
\end{tabular}

Table 9: Summary of the attained risk tolerances $(\tau=0.01$ and $c=2)$.

parameters $a$ and $\bar{\epsilon}$ are the two main levers for manipulating the reliability levels associated with the stochastic demand satisfaction constraints. In our analysis, we consider three benchmark models: $i$ ) the quantitative measure is excluded by setting $a=0$ with $\bar{\epsilon}=0.3$ (or $\bar{\epsilon}=0.5$ ) for the qualitative measure (Benchmark 1), ii) the qualitative measure is excluded by setting $\bar{\epsilon}=1$ with $a=100,000$ for the quantitative measure (Benchmark 2), $i i i$ ) both the quantitative and qualitative measures are excluded by setting $a=0$ and $\bar{\epsilon}=1$, respectively (Benchmark 3). In the second benchmark model, setting $\bar{\epsilon}=1$ requires us to resort to a more traditional approach of accounting for the demand shortages by measuring the shortage amount at node $k$ against the actual delivery amount $T_{k} \mathbf{x}$ instead of $\operatorname{VaR}_{\left(1-\epsilon_{k}\right)}\left(\xi_{k}\right)$. For all models involved, we compute the realizations of the maximum and average proportion of unsatisfied demand with respect to the delivery amounts - MPUD and APUD, respectively - across all demand points under each scenario. The resulting cumulative distribution functions (CDFs) are plotted in Figure 1. This figure illustrates that (CCSRND) outperforms all three benchmark models with respect to both performance measures MPUD and APUD. The curves associated with (CCSRND) always appear to the left of the rest, i.e., they are stochastically smaller than the others. For both (CCSRND) and Benchmark 1, enforcing more restrictive chance-constraints by increasing the value of $a$ or decreasing the value of $\bar{\epsilon}$ up to some threshold improves the reliability, and consequently, enhances the PUD-related performance measures. The interplay between $a$ and $\bar{\epsilon}$ is delicate; an overly restrictive value of one of these parameters may render the model insensitive to the changes in the other. This is evident from the CDFs associated with (CCSRND) for $a=10^{6}$ which are virtually identical for both values of $\bar{\epsilon}$. (CCSRND) with this setting of $a$ overemphasizes the quantitative measure in supply allocation and tips the trade-off in the objective function in disfavor of accessibility - as observed previously in Table 8. While this essentially promotes better PUD-related performance as illustrated in Figure 1, we do necessarily advocate it because it misses the point of balancing the trade-off between the total accessibility and the cost of reliability associated with the demand satisfaction in the objective function. The trade-off is captured much better by reducing $a$ to $10^{5}$. Then, increasing $\bar{\epsilon}$ from 0.3 to 0.5 leads to a right shift in the CDF associated with (CCSRND) for both MPUD and APUD, and we can also rely on $\bar{\epsilon}$ to strike a satisfactory performance level in terms of both accessibility and reliability. Among the three benchmark models, Benchmark 1 is closest to (CCSRND) in terms of MPUD. In the case of APUD, the ranking of Benchmarks 1 and 2 is determined by the value of $\bar{\epsilon}$ which impacts Benchmark 1 negatively. It is also fairly obvious that Benchmark 2 with a higher $a$ value would track (CCSRND) with $a=10^{6}$ pretty closely as indicated by the insensitivity of (CCSRND) with $a=10^{6}$ to different values of $\bar{\epsilon}$. In summary, we can confidently state that the presented benchmarking analysis provides sufficient evidence for the value of our proposed framework with the embedded hybrid supply allocation policy as a flexible modeling tool to balance the trade-off between the total accessibility and the cost of reliability.

One of the recurring themes in this section is that the attained risk tolerances, the cost of reliability, and the actual cost / return are sensitive to the values of the parameters $a$ and $\bar{\epsilon}-$ as expected. From a decision making viewpoint, the values of these parameters are an expression of the decision maker's qualitative and quantitative 


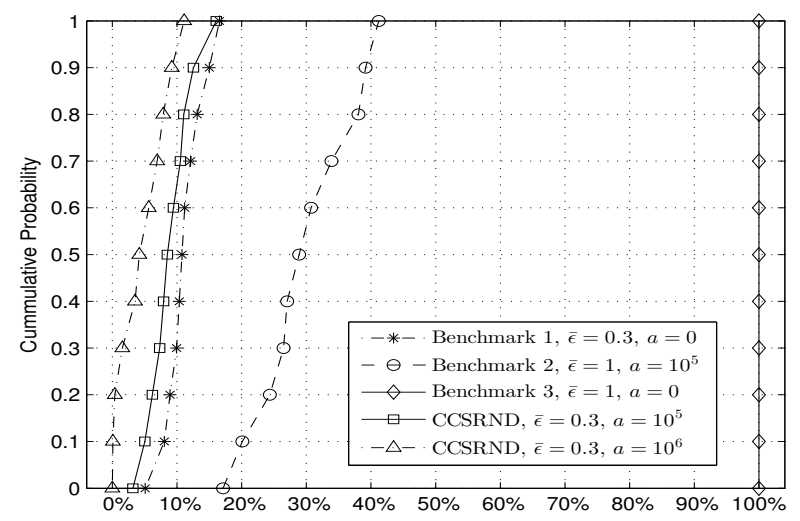

(a) $\operatorname{MPUD}(\bar{\epsilon}=0.30)$

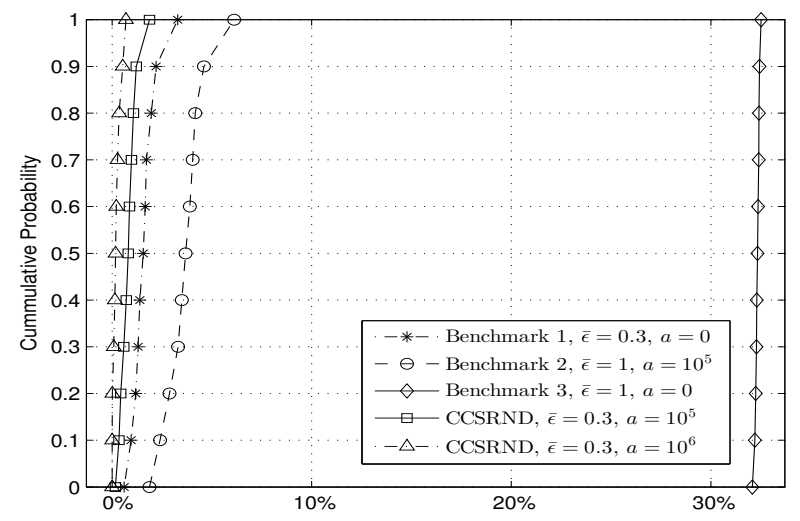

(c) $\operatorname{APUD}(\bar{\epsilon}=0.30)$

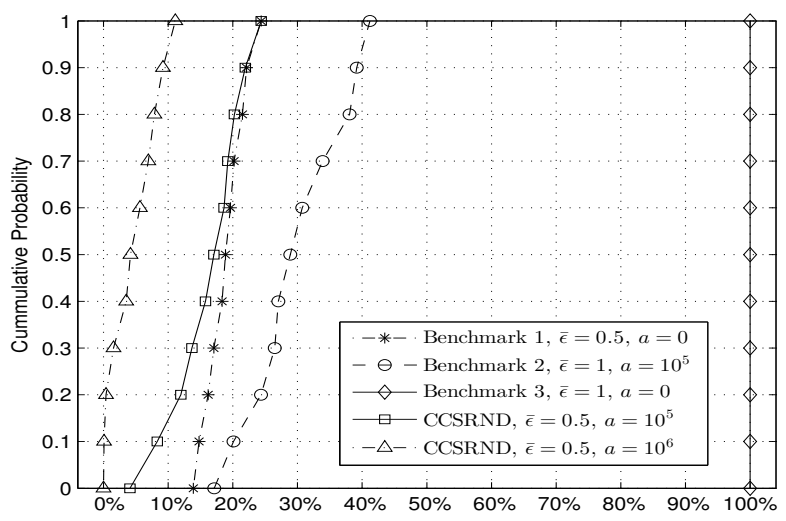

(b) $\operatorname{MPUD}(\bar{\epsilon}=0.50)$

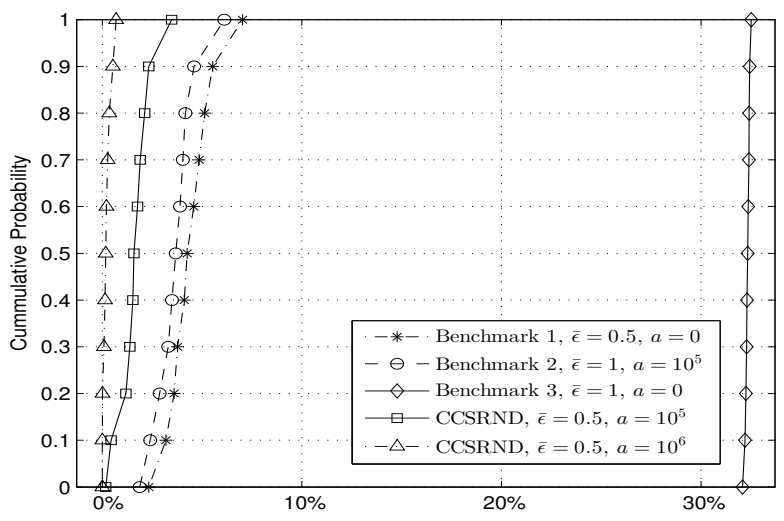

(d) $\operatorname{APUD}(\bar{\epsilon}=0.50)$

Figure 1: Empirical cumulative distribution functions of MPUD and APUD $(\tau=0.02, c=2.0)$.

risk preferences, and it is completely the decision maker's prerogative to determine the right values. However, the decision maker may not be entirely comfortable with setting a single value for $a$ or $\bar{\epsilon}$ and need assistance. To this end, we reckon that our models can be solved iteratively within a Pareto-type framework. More specifically, a grid of possible values for one or several $a$ or $\bar{\epsilon}$-parameters may be defined so that the attained reliability levels, the cost of reliability, and the actual cost/return are explored and plotted over this grid. This rationale is very much akin to how the Pareto frontier is constructed in multi-objective optimization by varying the scaling parameters in the weighted objective.

6. Conclusion In this paper, we develop strong MIP formulations for two classes of individual chanceconstrained linear programs with variable reliability levels / risk tolerances by leveraging recent methodological advances. A new type of cost function, which requires capturing VaR associated with a variable reliability level, is an additional contribution of our work. Our computational study attests to the effectiveness of the proposed MIP formulations in solving the problems of interest. Optimal solutions of large practical-size instances of these formulations are within the reach of modern off-the-shelf solvers. The application of the proposed models to a novel stochastic last mile distribution network design problem and the associated case study based on real-life data further substantiates this claim. We hope that our work will invoke more awareness about the availability of powerful modeling tools for individual chance-constrained optimization models with and without variable reliability levels. 
Acknowledgments Nilay Noyan was supported in part by The Scientific and Technological Research Council of Turkey (TUBITAK) Career Award \# 111M543 and by Bilim Akademisi - The Science Academy, Turkey, under the BAGEP program. Ozgun Elci was partially supported by the TUBITAK BIDEB program. The authors also acknowledge the support from TUBITAK under grant \# 112M864. The authors are sincerely grateful to J. Luedtke for providing the data sets for the probabilistic transportation problem, and also thank the anonymous referees for their valuable feedback.

\section{References}

Balcik, B. and Beamon, B. (2008). Facility location in humanitarian relief. International Journal of Logistics: Research and Applications, 11(2):101-121.

Beraldi, P. and Bruni, M. E. (2009). A probabilistic model applied to emergency service vehicle location. European Journal of Operational Research, 196(1):323-331.

Dentcheva, D. (2006). Probabilistic and Randomized Methods for Design under Uncertainty, chapter Optimization Models with Probabilistic Constraints. Springer-Verlag, London. Editors: G. Calafiore and F. Dabbene.

Döyen, A., Aras, N., and Barbarosoğlu, G. (2012). A two-echelon stochastic facility location model for humanitarian relief logistics. Optimization Letters, 6:1123-1145.

Elçi, O. and Noyan, N. (2018). A chance-constrained two-stage stochastic programming model for humanitarian relief network design. Transportation Research Part B: Methodological, 108:55-83.

Günlük, O. and Pochet, Y. (2001). Mixing mixed-integer inequalities. Mathematical Programming, 90(3):429457.

Haneveld, W. K. K. and van der Vlerk, M. H. (2015). Stochastic programming lecture notes. Available on request (only as hard copy; view Table of contents at http://www.rug.nl/feb/mhvandervlerk/spcourse/ contents.pdf.

Hong, X., Lejeune, M. A., and Noyan, N. (2015). Stochastic network design for disaster preparedness. IIE Transactions, 47(4):329-357.

Kahvecioglu, G. (2014). Stochastic last mile relief network design with resource reallocation. MS Thesis, Sabanci University.

Kall, P. and Wallace, S. (1994). Stochastic programming. Wiley-Interscience series in systems and optimization. Wiley.

Küçükyavuz, S. (2012). On mixing sets arising in chance-constrained programming. Mathematical Programming, 132(1-2):31-56.

Küçükyavuz, S. and Noyan, N. (2016). Cut generation for optimization problems with multivariate risk constraints. Mathematical Programming, 159(1):165-199.

Lejeune, M. and Noyan, N. (2010). Mathematical programming approaches for generating p-efficient points. European Journal of Operational Research, pages 590-600.

Lejeune, M. A. (2012). Pattern-based modeling and solution of probabilistically constrained optimization problems. Operations Research, 2(1):1356-1372.

Lejeune, M. A. and Shen, S. (2016). Multi-objective probabilistically constrained programs with variable risk: Models for multi-portfolio financial optimization. European Journal of Operational Research, 252(2):522 539 .

Liberatore, F., Pizarro, C., de Blas, C., Ortuño, M., and Vitoriano, B. (2013). Uncertainty in humanitarian logistics for disaster management. a review. In Vitoriano, B., Montero, J., and Ruan, D., editors, Decision Aid Models for Disaster Management and Emergencies, volume 7 of Atlantis Computational Intelligence Systems, pages 45-74. Atlantis Press.

Luedtke, J. (2014). A branch-and-cut decomposition algorithm for solving chance-constrained mathematical programs with finite support. Mathematical Programming, 146(1-2):219-244.

Luedtke, J., Shabbir, A., and Nemhauser, G. (2010). An integer programming approach for linear programs 
with probabilistic constraints. Mathematical Programming, 122(2):247-272.

Mete, H. O. and Zabinsky, Z. B. (2010). Stochastic optimization of medical supply location and distribution in disaster management. International Journal of Production Economics, 126(1):76-84.

Noyan, N. (2012). Risk-averse two-stage stochastic programming with an application to disaster management. Computers and Operations Research, 39(3):541-559.

Noyan, N., Balcik, B., and Atakan, S. (2016). A stochastic optimization model for designing last mile relief networks. Transportation Science, 50(3):1092-1113.

Prékopa, A. (1995). Stochastic Programming. Kluwer Academic, Dordrecht, Boston.

Prékopa, A. (2003). Handbooks in Operations Research and Management Science, vol.10, chapter Probabilistic Programming, pages 267-351. Elsevier, Amsterdam. Editors: A. Ruszczyński and A. Shapiro.

Rawls, C. G. and Turnquist, M. A. (2010). Pre-positioning of emergency supplies for disaster response. Transportation Research Part B: Methodological, 44(4):521-534.

Rawls, C. G. and Turnquist, M. A. (2011). Pre-positioning planning for emergency response with service quality constraints. OR Spectrum, 33(3):481-198.

Rengarajan, T., Dimitrov, N., and Morton, D. (2013). Convex approximations of a probabilistic bicriteria model with disruptions. INFORMS Journal on Computing, 25(1):147-160.

Rengarajan, T. and Morton, D. (2009). Estimating the efficient frontier of a probabilistic bicriteria model. In Proceedings of the 2009 Winter Simulation Conference (WSC), pages 494-504.

Ruszczyński, A. (2002). Probabilistic programming with discrete distributions and precedence constrained knapsack polyhedra. Mathematical Programming, 93(2):195-215.

Salmerón, J. and Apte, A. (2010). Stochastic optimization for natural disaster asset prepositioning. Production and Operations Management, 19(5):561-574.

Shapiro, A., Dentcheva, D., and Ruszczyński, A. (2009). Lectures on stochastic programming: modeling and theory. The society for industrial and applied mathematics and the mathematical programming society, Philadelphia, USA.

Shen, S. (2014). Using integer programming for balancing return and risk in problems with individual chance constraints. Computers \&6 Operations Research, 49:59 - 70.

Xie, W., Ahmed, S., and Jiang, R. (2017). Optimized Bonferroni approximations of distributionally robust joint chance constraints. http://www.optimization-online.org/DB_HTML/2017/02/5860.html. 